\title{
Functional equivalence revisited: Costs and benefits of priming action with motor imagery and motor preparation
}

\author{
Benjamin Robert William Toovey ${ }^{1,2}$, Ellen Seiss $^{3}$, Annette Sterr $^{2,4}$ \\ ${ }^{1}$ Martin-Luther University of Halle-Wittenberg, Halle, Germany \\ ${ }^{2}$ University of Surrey, Guildford, UK \\ ${ }^{3}$ Bournemouth University, Bournemouth, UK \\ ${ }^{4}$ Center for Post-Acute Neurorehabilitation, Berlin, Germany
}

The authors have no competing interests to declare. BRWT was supported by a FAHS Faculty Studentship from the University of Surrey, Guildford, UK.

Word Count: 11502

Correspondence to the authors:

Dr. Benjamin Toovey

brwtoovey@googlemail.com

Martin-Luther-Universität Halle-Wittenberg

Institut für Psychologie

06099 Halle (Saale)

Tel: +49(0)345 5524371

Fax: +49(0)345 5527060

OSF repository: https://osf.io/mruxp/

(C) 2021, American Psychological Association. This paper is not the copy of record and may not exactly replicate the final, authoritative version of the article. Please do not copy or cite without authors' permission. The final article will be available, upon publication, via its DOI: $10.1037 / \mathbf{x h p 0 0 0 0 9 6 6}$ 


\begin{abstract}
The functional equivalence (FE) hypothesis suggests motor imagery (MI) is comparable with the planning stages of action. A strong interpretation of this hypothesis suggests MI can prime subsequent actions in a way that should be indistinguishable from motor preparation (MP). Alternatively, MI could involve more richly informative motor plans than MP, producing different effects on the performance of subsequent actions. Although past research has demonstrated MI can prime action, little research directly compared it to MP, thus the value of strong FE interpretations for understanding MI remains unclear. In the present study, a pre-cueing paradigm was used in four experiments, and congruency effects were compared. Precues could either prepare participants for specific responses (MP condition) or participants should imagine the precued response (MI condition), prior to making their response. Experiment 1 provided first evidence favouring our alternative hypothesis that predicted imagery-primed responses result in larger response priming effects than prepared-responses, meaning that it might involve more richly informative motor plans than preparation for action. Experiment 2 manipulated precue - imperative stimulus foreperiods and replicated this effect, showing it to be independent of potential temporal uncertainty differences between the two conditions. Experiment 3 showed the effect is present in both foot and finger responses, and experiment 4 suggested the larger congruency effects in the MI condition cannot be explained by differences in cognitive load. These results suggest that the strong FE hypothesis does not hold. Findings are discussed in line with the predictive processing models of action and MI.
\end{abstract}

Public Significance Statement: Motor imagery is a promising tool for neurorehabilitation, so a better understanding of how motor imagery works is becoming increasingly important as a result. This work shows that motor imagery can influence subsequent action to a greater extent than preparation for action alone. This evidence provides a new and meaningful development to the functional equivalence hypothesis.

Keywords: motor imagery; motor preparation; neural simulation; functional equivalence; response priming 


\section{Functional equivalence revisited: Costs and benefits of priming action with motor imagery and motor preparation}

The ability for people to imagine their own actions, or motor imagery (MI), is a behaviour frequently used to investigate the representation and control of action in humans. The functional equivalency hypothesis (hereafter $\mathrm{FE}$ ) proposes that $\mathrm{MI}$ is more similar to motor preparation (MP) preceding action (Jeannerod, 1994; Hanakawa, 2016) than with action itself, as evidenced in recent meta-reviews of fMRI research on the topic (Hétu et al, 2013; Hardwick et al, 2018). Although this is a useful heuristic for researchers investigating commonalities between MI and action proper, FE and the related neural simulation theory (Jeannerod, 2001) may be unsuitable for understanding mechanisms that are unique to MI (O’Shea \& Moran, 2017; Glover \& Baran, 2017). Accumulated behavioural and neural evidence demonstrates differences between MI and action which reflects this problem (Hanakawa et al, 2008; Kranczioch et al, 2009; 2010; Lebon et al, 2019; Ingram et al, 2016; Solomon et al, 2019; Glover et al, 2020). To examine the FE hypothesis in more detail, in this paper we present a series of behavioural experiments which directly compare the effects of MI and MP on priming subsequent actions in an S1-S2 response priming paradigm.

Prior to the initiation of cued or voluntary action, a person can plan one or several specific movement parameters (Cisek \& Kalaska, 2005; Rosenbaum, 1980). These parameters can be defined in terms of information about "what" (action to perform), "when" (to perform it), and "whether" (to perform an action at all; Brass \& Haggard, 2008). Importantly, advanced knowledge of these parameters is reflected in both behavioural (Rosenbaum 1980; Brown et al, 2011) and electrophysiological metrics (Leuthold et al, 2004; Korka et al, 2021). For instance, explicit advanced specification of full rather than only partial "what" information reduces reaction times and increases accuracy of the upcoming action (Rosenbaum, 1980). Moreover, the amplitude of the lateralised readiness potential (LRP), a neural marker for MP, is greater as a function of the amount of explicit advanced "what" information cued for participants prior to subsequent movements (Leuthold et al, 2004), or when voluntary button presses are associated with specific versus unspecific action-effects (Korka et al, 2021). 
Under a strong interpretation of the FE hypothesis, MI should reflect the quality and reliability of motor plans in a similar way to MP, as described above. Alternatively, while MI may involve the same underlying motor plans as MP, MI could be more informative for subsequent behaviour. Indeed, this was alluded to in Jeannerod's original 1994 paper, in which he suggested a continuum between MP and MI - although it was suggested this is dependent on temporal differences rather than differences in relative informational content of the underlying motor plans themselves. This alternative hypothesis captures a key introspective difference between MI and MP - imagining oneself grasp the handle of a coffee cup is a "richer" experience than simply preparing to make the same movement. This suggestion is supported by prior research showing that, even without extensive training regimes, real performance is sensitive to a period of immediately preceding MI on a trial-bytrial basis (Ramsey et al, 2010; Li et al, 2004, 2009; Anwar et al, 2011; Jeannerod, 1994). For instance, in the study of Anwar et al, (2011), participants were first trained to make directionally cued physical reaching movements in a motion-perturbing forcefield. In a subsequent testing session, participants either imagined or prepared their reaches prior to the action. On catch trials, the forcefield was removed unexpectedly. Results showed that only on catch trials with preceding MI, participants showed substantial deviations in their reach trajectories, even though no compensatory force was required. In contrast these unnecessary deviations were absent on catch trials with preceding MP. These data suggest MI may indeed involve more richly informative motor plans than MP alone, and that these plans are continuously available until the subsequent action is performed.

If MI plans are more informative for subsequent action relative to MP plans, one would also expect to see large behavioural priming effects following MI. This was investigated by Ramsey and colleagues (2010). Here, participants were asked to perform a reaching action towards a physical target object after first imagining a target reach that was either congruent or incongruent with the real reaching action. When compared to a non-MI baseline condition, movement initiation times were prolonged following incongruent MI reaching, but not accelerated following congruent MI reaching. The authors concluded that there were interference effects, but not benefits of MI on subsequent action. These findings suggest, similar to Anwar et al, (2011), that reaching actions can integrate 
motor plan information generated during MI, as would be expected by the FE hypothesis. However, the researchers observed only interference effects. This suggests that there are limits to the degree to which FE holds between MI and MP, since both facilitation and cost effects on action should be expected for congruent and incongruent MI conditions, as has been previously reported in response priming tasks without MI conditions (Leuthold, 2003).

Note, however, that Ramsey et al, (2010) did not explicitly compare MI and MP in their study, rather only MI and no-MI. Therefore, it remains unclear whether MI and MP would have produced similar patterns of priming effects on subsequent action within the same experiment. This is an important comparison, theoretically speaking, because the FE in its original formulation cannot distinguish priming effects of MI and MP - indeed it suggests similar effects, and merely that (unconscious) MP can become (conscious) MI when extended over longer time periods (Jeannerod, 1994). This means it is unclear whether the MI priming effects reported by Ramsey et al, (2010; see also Li et al, 2004; 2009) cannot be more simply explained by assuming participants were engaged in MP alone. The question of whether MI and MP priming effects diverge within the same paradigm, has remained relatively untested. Hence, we propose that a direct comparison of MI and MP priming effects is still required, as this will be more informative about the nature of MI as it relates to MP under a strong FE hypothesis.

When examining MI effects, it is important to select an appropriate baseline condition. In Ramsey et al, (2010), comparing MI reaches with a subsequent congruent or incongruent real reach against a no-MI priming baseline condition may indeed reflect priming effects of MI, but there is an alternative interpretation of these findings. During MI, the absence of overt movement has been attributed to a variety of inhibitory signals, which operate at global or effector-specific levels (Guillot et al, 2012a), persisting over time (Rieger et al, 2017; Bart et al, 2020), and possibly as an inherent element of the motor plan itself (Eagles et al, 2015; Solomon et al, 2019). Since inhibition produced during MI would need to be overcome first, response initiation would be delayed in both incongruent and congruent conditions relative to no-MI, where no prior inhibition of the motor plan occurs. Therefore, an unaccounted-for inhibitory element of MI may have cancelled any facilitation and 
inflated the observed interference effects (Ramsey et al, 2010; see also Li et al, 2004; 2009). Thus, a direct comparison between unadjusted reaction or movement initiation times in MI and MP may be unsuitable for drawing insight about how the two behaviours compare in terms of their motor priming effects. Alternatively, we propose that directly comparing differences in priming effects (i.e. incongruent response times $(\mathrm{RT})$ - congruent $\mathrm{RT}$ ) provides a better analytical logic to examine the similarities or differences between the MI and MP conditions. Since priming effects are computed by subtraction, any MI-related inhibition, which will be present in both congruent and incongruent MI trials, is accounted for when computing them. This subtractive logic accounts for any processes that are present for all trial types, but which are unrelated to the relative effects of motor priming, such as inhibition. Subsequently, these priming effects can be compared across MI and MP conditions. To further refine the examination of priming effects due to MI and MP, a neutral condition for which only advanced temporal ("when") but no identity ("what") information can be introduced to disentangle subcomponents of primed (intentional) action which contribute to MI-based priming effects. Similarly as in Posner (1980), this neutral condition can be used to fractionate the overall congruency effect into the relative cost and benefit effects in both MI and MP. By examining these difference scores instead of unadjusted reaction or initiation time, we can characterise more accurately the facilitative and/or interfering nature of MI relative to MP on subsequent performance, and examine whether or not MI priming effects are indeed larger compared to MP alone.

\section{Experiment 1}

In the present study, we aimed to test how MI priming influences subsequent action, and whether it can indeed be distinguished from MP, by directly comparing MI and MP effects on subsequent actions using a response priming paradigm (Rosenbaum, 1980; Leuthold et al, 2003; Brown et al, 2011). Participants were instructed to respond to a visual imperative stimulus using their feet. In separate sets of blocks, these responses were preceded by a visual precue stimulus, which required participants to either imagine or prepare a corresponding foot movement during the interstimulus foreperiod. To account for both beneficial and costly effects of MI on subsequent action, and to differentiate these from the effects of MP while also controlling for baseline differences such as 
additional MI-related inhibition, the present experiment manipulated precue validity to create real responses which were congruent or incongruent with the preceding imagined or prepared action. An additional neutral priming condition was introduced, which allowed for a comparison of the magnitude of the mean costly and beneficial priming effects between MI and MP. Foot movements were used as the main method of response and imagery throughout this experiment because lowerlimb movements are under-represented relative to upper limb movements in MI research (Hétu et al, 2013), and participants' imagery ability was also assessed using the kinesthetic and visual scales of the MIQ-RS (Gregg et al, 2010).

In experiment 1 (and all other experiments in this paper), we calculated the overall congruency effect (CE) by subtracting mean RTs on congruent from incongruent trials. This was the primary dependent variable. To examine relative effects of congruently versus incongruently primed responses, the CE was subdivided into benefits by subtracting mean RT on congruent from neutral trials, and costs by subtracting mean RT on neutral from incongruent trials. A similar analysis was conducted for mean error rates, computed as percentages of incorrect responses for MI and MP trial types.

If MI involves more informative motor plans compared to MP alone then we expected the magnitude of the overall CE to be larger in the MI than in the MP condition. We also expected both MI and MP could facilitate and interfere with responses relative to neutral priming, and thus both would elicit benefit and cost effects respectively. To examine this, we compared MI and MP conditions on their cost and benefit magnitudes. This fractionation of the $\mathrm{CE}$ into costs and benefits serves to indicate whether any differences in the overall CE between MI and MP can be attributed to differences in facilitation (e.g., larger benefits in MI) versus interference (e.g., larger costs in MI), or whether the balance of subcomponents is different between the two conditions even if the overall CE is numerically equivalent (e.g. larger benefits but smaller costs in MI). Finally, to examine whether there were any overall differences between MI and MP associated with general inhibitory effects (Guillot et al, 2012a), we compared the unadjusted neutral trial RTs between MI and MP conditions. This is because neutral trials should involve non-specific response priming in both conditions, but 
would be associated with MI-related inhibitory processes in MI, and the absence of such inhibitory processes in MP. For this analysis, we anticipated slower neutral trial type RTs in MI compared to MP conditions.

\section{Method}

\section{Participants}

Twenty individuals recruited from the University of Surrey took part in this experiment. They provided informed consent and were rewarded with course credits. One participant's dataset was removed from analysis due to having slow RTs in at least two trial types (more than 2.5 SD of the group mean). The final sample consisted of nineteen individuals, aged $19-29$ years old (Mean age $=$ 22.4 years; 18 females, 1 male). All participants reported normal or corrected-to-normal vision and colour vision. Handedness was assessed using the Edinburgh Handedness Inventory (EHI: Oldfield, 1971), with all participants reporting as being dominant right-handed. A single question ("Which is your dominant foot?") assessed foot dominance. One individual was left foot dominant, twelve were right foot, and five were neither left nor right foot dominant. The experiment was conducted in accordance with the principles of the Declaration of Helsinki and received local institutional ethical approval from the University of Surrey.

To evaluate the power of our sample to detect a certain effect size, we computed a sensitivity analysis using G*Power 3.1 (Faul et al, 2009) based on a two-tailed dependent-measures t-test comparing the congruency effects for MI and MP conditions. With a sample size of 19 and a power of .80, this experimental design was sufficiently powered to reliably detect a Cohen's dz (t/sqrt(n); see Lakens, 2013) of 0.68 for this critical comparison. A sensitivity analysis reflects the minimum reliable effect size that can be obtained based on the sample size provided.

\section{Stimuli, Material and Equipment}

Precue (S1) stimuli were brown circles subtending $5.9^{\circ}$ x $5.7^{\circ}$ degrees of visual angle. One half of the circle was filled black. Imperative (S2) stimuli were white semicircles subtending $3.2^{\circ} \mathrm{x}$ 
$5.7^{\circ}$ degrees of visual angle. Two keyboard sustain pedals (Cherub WTB-004) were used as response buttons. A switch on their side allowed participants to register responses with either (a "lift" or a "press" response). The experiment was presented on a 19-inch CRT monitor, running on a desktop computer with Windows 7 operating system, programmed and delivered using Presentation (Version 16.4, Neurobehavioural Systems). Participants were seated $100 \mathrm{~cm}$ from the screen.

The MIQ-RS (Gregg et al, 2010) is a questionnaire designed to assess self-reported kinesthetic and visual imagery ability. Each scale contained 7 questions referring either to the kinesthetic $(\mathrm{k})$ properties or vividness $(\mathrm{v})$ of a motor or visual motor imagined movement respectively, rated on a scale of 1 to 7 . (1= Very hard to feel $(\mathrm{k}) / \mathrm{see}(\mathrm{v}) ; 7=$ Very easy to feel $(\mathrm{k}) /$ see (v)). This data was recorded to ensure participants could sufficiently imagine their actions in a kinaesthetically, but the data is not reported within this paper for brevity.

\section{Task and Procedure}

Prior to commencing the experiment, participants provided signed informed consent, and were administered the MIQ-RS. Before the first block-set (consecutive blocks of trials with instructions for MI or MP - see below), participants moved the foot pedals to a position under the desk to a comfortable position.

In the experimental task, all stimuli were presented on a black background. They were positioned centrally on the computer monitor. Each trial began with a white fixation cross for $500 \mathrm{~ms}$, which was replaced by S1 for $150 \mathrm{~ms}$. After S1-offset, the fixation cross re-appeared, and lasted for $3350 \mathrm{~ms}$ (the "foreperiod"). At the end of the foreperiod, S2 replaced the fixation cross for $150 \mathrm{~ms}$, which was followed by a blank screen in which participants were required to make a left or right foot response, depending on the identity of S2. An intertrial interval of $700 \mathrm{~ms}$ was presented before the next trial began.

To create congruent, incongruent, and neutral priming effects, the brown half of the S1 stimulus could point to the left or to the right (informative S1), or it could be oriented downwards (uninformative S1). Informative and uninformative precues were presented in a ratio of 2:1. The 
orientation of S1 dictated which response should be prepared or imagined prior to S2. When the brown half of S1 pointed to the left or right of the screen, participants would imagine or prepare a left or right foot response during the foreperiod, depending on block-set instructions, respectively. When it pointed to the bottom of the screen, they prepared for either a left or right foot response to S2 or imagined both feet moving in synchrony during the foreperiod. The orientation of the white half of the S2 stimulus dictated the required response - pointing to the left or right of the screen required a left or right foot response, respectively. An informative S1 congruently predicted S2 in $80 \%$ of cases, and incongruently in $20 \%$ of cases. An uninformative S1 indicated a 50\% chance of making a left or right foot response to S2. Across all trials, correct responses were required in equal proportion for left and right foot. Participants were fully informed about the congruency manipulation and proportions prior to beginning the experiment.

Each block-set contained four blocks of 60 trials. Each block contained 32 congruent, 20 neutral, and 8 incongruent primed trials. At the beginning of each block set, participants practiced 12 trials, two from each congruency manipulation per foot, and would progress to the main block only with $80 \%$ accuracy. Participants were allowed short breaks between blocks, and between block-sets while new instructions were presented and read out to participants.

In the MP block-set, participants were instructed to prepare fast and accurate responses to S2, based on the information provided by S1. For example, a leftward or rightward informative S1 would require participants to prepare to make a left or right foot response following S2. In trials with a neutral (uninformative) S1, participants were informed that a left or right foot response would be required with equal probability by the $\mathrm{S} 2$, and that they should thus prepare for both alternatives (Posner, 1980; Cisek \& Kalaska, 2005). In the MI block-set, participants were instructed to make three imagined consecutive foot movements, like exaggerated button presses, during the foreperiod, at a rate of once per second. Left and right S1 required left and right foot imagery, during the foreperiod respectively. Neutral S1 required bipedal synchronous foot imagery, to engage MI performance without providing specific advanced information about which effector will be required for responding. Participants were asked to perform the imagined movements from a first-person perspective (thereby 
engaging kinesthetic as opposed to visual imagery; Lorey et al, 2009), to focus on creating and feeling the motions and sensations associated with real movements, and to restrict themselves from making any physical movement until S2. Instructions for MI or MP were provided on-screen and verbally prior to the relevant block-set.

All block-sets with instructions for participants to engage in MP during the S1-S2 foreperiod always preceded block-sets with instructions to perform MI. Despite potential order-effect issues regarding the fixed order of MP and MI block-sets, we viewed it more problematic that counterbalancing the order of MP and MI block-sets would allow the possibility for participants to use the specific strategies we instructed for MI performance during MP block-sets. In this case, we would be unable to control MI behaviours from percolated into, and thereby confounding, MP block-set trials.

\section{Figure 1}


1A: Trial Schematic

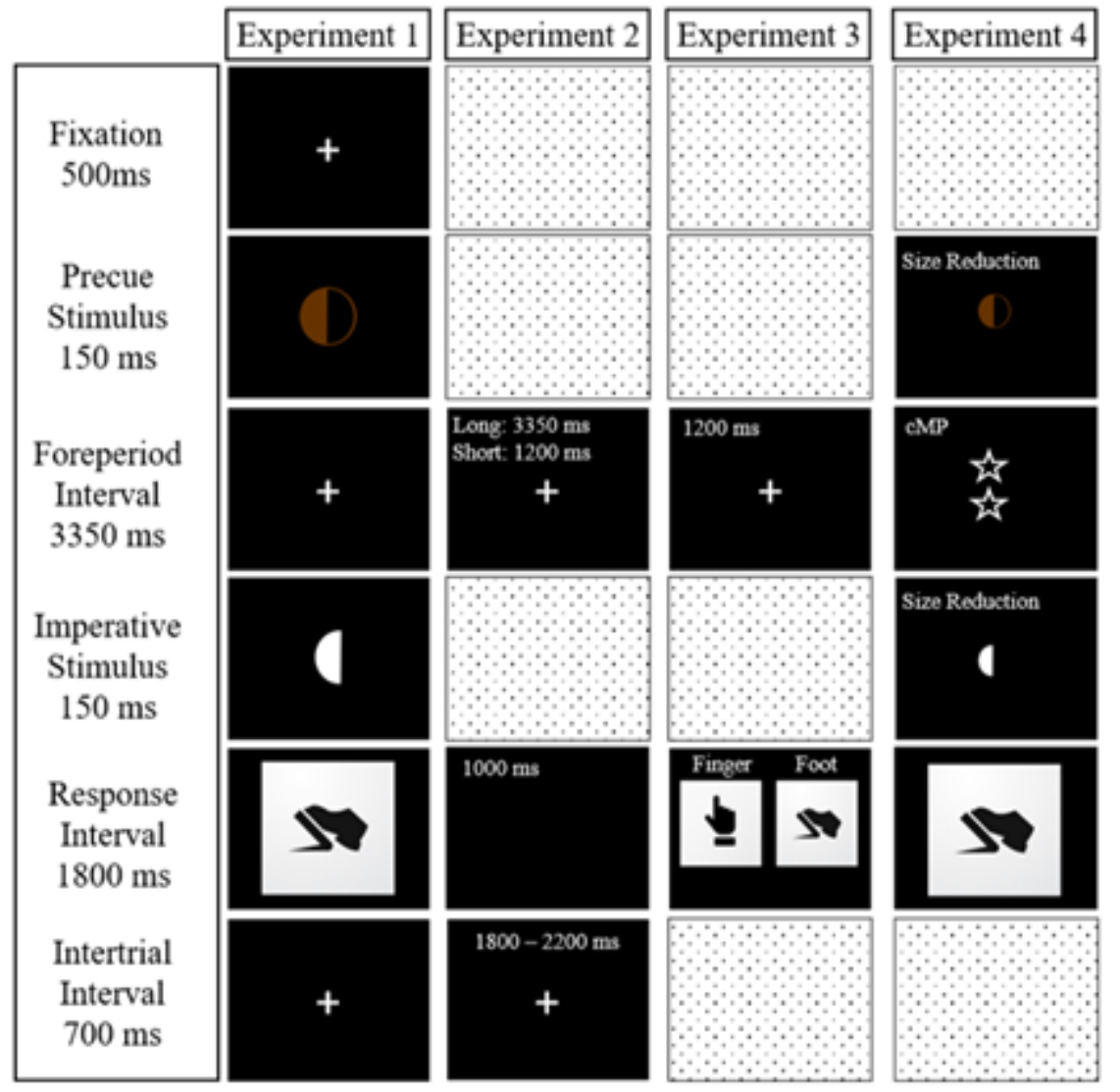

1B: Trial Proportions and Validity Relationships

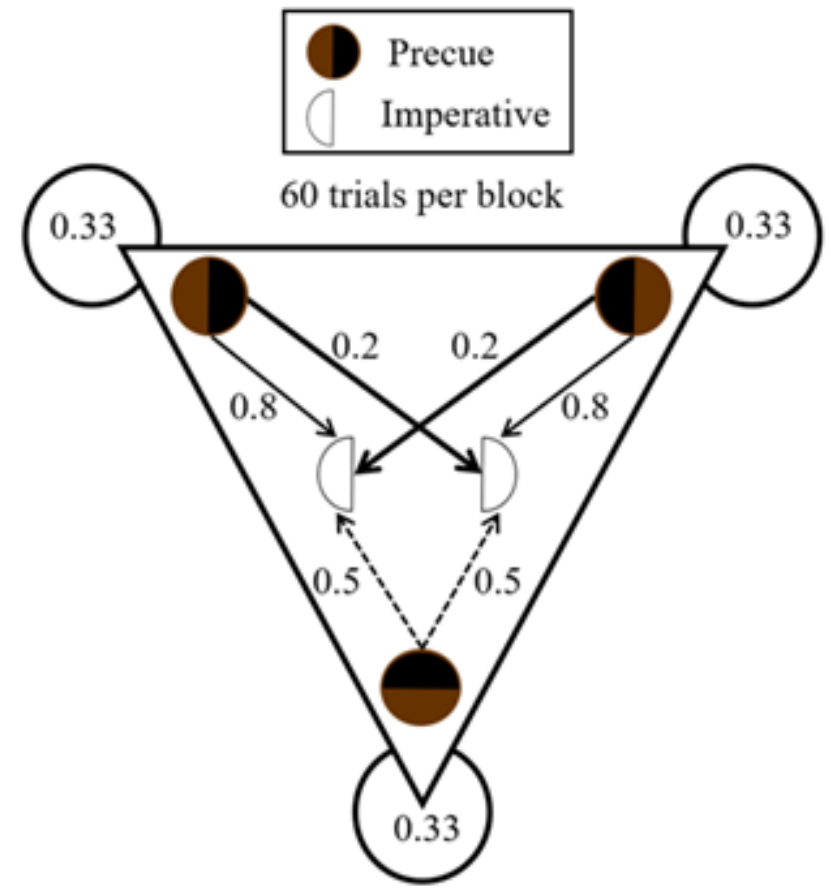


Figure 1. An overview of the trial design in each experiment. In Figure 1A, trial schematics for each experiment are displayed. Grey dotted cells represent continuity in design between experiments, and the filled cells represent the elements that change. In Figure 1B, the diagram shows the relative proportions of trial types within a block, as well as the relationships between precue and imperative stimuli. 


\section{Design and Analysis}

Practice trial data were not analysed. Trials with incorrect responses, or with RTs faster than $100 \mathrm{~ms}$ were not included in the RT analysis (data removed in $\mathrm{MI}=1.91 \%, \mathrm{MP}=1.53 \%$ ). First, mean neutral trial RTs were compared between tasks, to see whether MI was slower than MP. Next, congruency, benefit and cost priming effects were computed as previously described, and these were also compared between MI and MP conditions. All tests were two-tailed paired t-tests. Full outputs comparing mean RTs for all trial types in both conditions are presented in Supplementary File 1. Data were pre-processed using Microsoft Excel and analysed using SPSS. Datafiles for all experiments in this paper can be found at https://osf.io/mruxp/.

\section{Results}

\section{Imagery Ability}

Participants mean kinesthetic imagery ability score was $5.34 \pm 0.94$, and visual score was $5.72 \pm 0.68$.

\section{Reaction Time Analysis}

Figure 2A shows the mean reaction times for congruent, neutral, and incongruent trial types and for each MI and MP conditions, separately. 


\section{Figure 2}

A: Mean reaction times ( $m s)$ for each trial type (congruent (Con), neutral (Neu), incongruent $(\mathrm{Inc})$ ) separated for the motor imaginary (MI) and motor preparation (MP) conditions. B: Violin plots to visualise individual RT differences in the distribution of congruency effects $(C E)$ for each condition. Dot and triangle markers represent individual participants. Dashed lines represent participants with an enhanced CEs for the MI compared to the MP condition. Solid lines represent a reduced CE in the MI compared to the MP condition. C: Mean cost and benefit effects (ms) for the MI and MP conditions. D: Mean error rates (\%) for each trial type (congruent, neutral, incongruent) separated for the MI and MP conditions. Error bars represent $\pm l$ SEM.
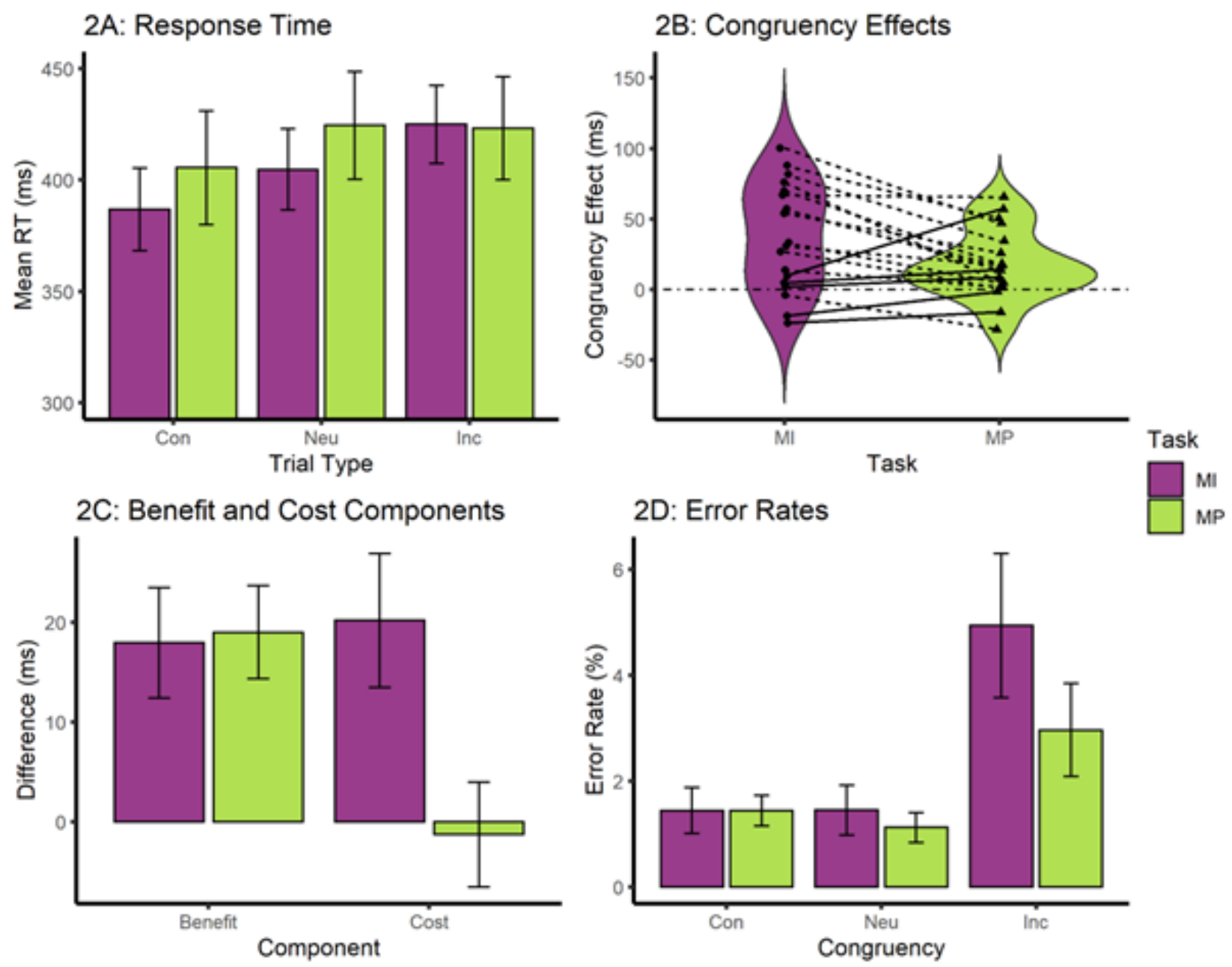


\section{Neutral RTs}

Reaction times in the neutral condition were not significantly slower in the MI compared to the MP condition (MI: $405 \pm 18.26 \mathrm{~ms}$; MP: $424 \pm 24.11 \mathrm{~ms}$; t(18)=1.453, p=.163).

\section{Congruency Effect}

Figure 2A shows mean RTs for the congruent, neutral and incongruent conditions.

Congruency effects (difference between congruent and incongruent conditions) were significantly larger in the MI compared to the MP condition (MI $=38 \pm 8.62 \mathrm{~ms}$ vs. MP $=18 \pm 5.59 \mathrm{~ms}$, difference: $20 \pm 6.76 \mathrm{~ms} ; \mathrm{t}(18)=3.016, \mathrm{p}=.007, \mathrm{dz}=0.69)$, showing stronger overall priming in MI compared to MP. These larger congruency effects in MI compared to the MP was found for most participants (Figure 2B).

\section{Benefit and Cost}

Figure 2C shows benefit (difference between congruent and neutral condition) and cost effects (difference between neutral and incongruent condition) for both MI and MP, but costs only in MI. Importantly, benefits did not differ significantly between the MI and MP conditions, $(\mathrm{MI}=18 \pm$ $5.53 \mathrm{~ms}$ vs. MP $19 \pm 4.65 \mathrm{~ms}$, difference: $1 \pm 6.17 \mathrm{~ms}$; $\mathrm{t}<1$ ). Costs, on the other hand, were larger for the MI compared to the MP condition $(\mathrm{MI}=20 \pm 6.71 \mathrm{~ms}$ vs. $\mathrm{MP}=-1 \pm 5.26$, difference: $21 \pm 7.29$ ms; $t(18)=2.943, p=.009)$. In summary, the enhanced congruency effects in the MI compared to the MP condition were driven entirely by enhanced cost effects in the MI condition.

\section{Error Rate Analysis}

In the error analysis (Figure 2D), neutral trials did not differ between MI and MP conditions (MI: $1.45 \pm 0.47 \%$; MP: $1.12 \pm 0.29 \% ; \mathrm{t}(18)=1.01, \mathrm{p}=.326)$. Congruency effects were significant for both the MI condition $(3.49 \pm 1.11 \% ; \mathrm{t}(18)=3.158, \mathrm{p}=.005)$ and the MP condition $(1.52 \pm 0.69 \%$; $t(18)=2.187, p=.042)$. However, there were no significant congruency effect, benefit or costs differences between the MI and MP conditions $(\mathrm{t}(18) \leq 1.687, \mathrm{p} \geq .109)$. 


\section{Discussion}

In experiment 1 , we observed faster responses and fewer errors for congruently primed trials than incongruently primed trials in both MI and MP instruction conditions. This suggests MI and MP can both prime subsequent action, as anticipated by the FE hypothesis (Jeannerod, 1994; Hanakawa, 2016). However, the overall congruency effects were larger for responses primed by MI than MP. This is in line with our proposal that MI priming may be distinguished from MP priming on the basis of the magnitude of overall priming effects. Moreover, this effect was entirely driven by a larger cost effect in MI, whereas the benefit effect was of a similar size in both conditions. The magnitude of the cost effect in the MP condition was, unexpectedly, negligible. Finally, there was no MI and MP condition difference for neutral trials, providing no strong evidence that inhibition differences between conditions can completely explain prior MI priming effects (e.g., Ramsey et al, 2010).

These results are broadly in line with previous studies suggesting MI can prime subsequent action (Ramsey et al, 2010; Li et al, 2004; 2009; Anwar et al, 2011), but it extends them in two important ways. First, they directly compare MI with MP priming effects and show that, in contrast to a strong FE hypothesis (Jeannerod, 1994), MI and MP can be distinguished on the basis of their magnitude of influence on subsequent actions. Second, they demonstrate facilitation as well as interference effects for MI priming. This was observed while controlling for baseline differences in the two conditions of interest which may have masked previous facilitation effects or enhanced interference (e.g., Ramsey et al, 2010). However, the absent cost effect was unexpected for the MP condition and may be an anomalous result which gave the appearance of a larger MI priming effect, rather than a true one. This finding requires additional clarification and, importantly, replication.

In order to further examine this initial result, three additional experiments were designed and conducted. Experiment 2 was designed to examine whether differences in temporal estimation between MI and MP contributed to the MI priming effects. In contingent response paradigms, longer foreperiods produce longer RTs than shorter foreperiods, an effect which has been ascribed to increased temporal uncertainty (Niemi \& Näätänen, 1981). It is possible that MI provides additional temporal certainty in longer foreperiods (a more general increase in "when" information; Brass \& 
Haggard, 2008), thereby increasing the magnitude of priming effects. To examine whether the larger MI priming effect depends on such optimal temporal preparation, we manipulated the length of the foreperiod blockwise to include a new, shorter foreperiod of $1200 \mathrm{~ms}$. In experiment 3, we addressed the novelty of lower limb responses and compared them to more commonly used upper limb (index finger) responses. Our day-to-day interactions require more conscious attention to the manipulation and positioning of our hands than our feet. Thus, the relative novelty of foot responses may have created an artefactually larger MI priming effect, whereby the imagination of foot movements interfered more strongly with subsequent performance, especially in incongruent trials. Therefore, we included a second set of blocks with finger responses, and preparation and imagery instructions. In experiment 4, we addressed the possibility that the MI priming effect was due to additional executive control requirements during MI trials. Given the recent evidence that MI performance relies on a limited pool of executive resources (Glover \& Baran, 2017), it is possible that this resource requirement, or additional motor-cognitive load, disrupts ongoing MP and artificially inflates the priming effects in MI blocks. In experiment 4 a third set of blocks was introduced which used normal MP task instructions, but participants were also required to perform a mental counting task during the foreperiod to enhance non-motor cognitive load. By comparing the non-motor cognitive load (MP plus counting control task) with the motor-cognitive load (MI task), we could evaluate this motorcognitive load explanation of the enhanced MI priming effects relative to MP.

\section{Experiment 2}

As stated above, the aim of experiment 2 was to investigate whether the larger MI priming effect depends on an optimal temporal preparation. We manipulated the length of the foreperiod blockwise to include a new, shorter foreperiod of $1200 \mathrm{~ms}$, and the foreperiod interval between S1 and S2 was manipulated blockwise. "Long" foreperiod blocks retained the same foreperiod duration as used in Experiment 1 (3350 ms), and were contrasted with the newer "short" foreperiod blocks. In short MI blocks, participants were instructed to make only a single imagined foot response during the foreperiod, rather than three as in long foreperiod blocks. Additionally, we suspected the absent MP cost effect in Experiment 1 may have been due to participants being less engaged with the task as 
compared with MI blocks. To encourage participants to engage more with the task and produce a significant cost effect in the MP task, we replaced the fixed intertrial interval with a jittered one in both MI and MP task blocks. Observing a significant cost in the MP task is an important goal, since it remains a plausible alternative explanation for the larger MI priming effect in Experiment 1.

We expected that longer overall RTs would be present in long foreperiod blocks, according to prior research (Niemi \& Näätänen, 1981). We also expected to observe an MI priming effect in both long and short foreperiods, and once again that this effect should be larger in MI than MP. If the MI priming effect would not depend on optimal temporal preparation, we expected there to be no interaction of its size with foreperiod duration. On the other hand, such an interaction would indicate MI priming does depend on such cognitive factors. More precisely, one possible interaction effect is that the MI priming effect will be larger than in MP for long than short foreperiods. This is because MI priming could reduce temporal uncertainty over long foreperiods relative to MP. In contrast, at short foreperiods temporal uncertainty would be generally greater in both conditions, and thus differences in MI or MP priming effects should be smaller or absent.

\section{Method}

\section{Participants}

Seventeen individuals aged $18-28$ years old (Mean age $=23$ years; 16 female, 1 male), who had not participated in experiment 1 , consented to participate in this experiment. One individual was left-handed, according to the EHI, 2 participants were left foot dominant, the remaining were right foot dominant. No participant datasets were removed due to slow responding (mean RT greater than 2.5 standard deviations from the group mean in at least four trial types).

A sensitivity analysis was conducted for the critical comparison between MI and MP congruency effects, reflecting a two-tailed paired t-test, a power of .80 and a sample size of 17 . With these parameters our experiment was sufficiently powered to detect effect sizes of Cohen's dz of 0.72 , assuming a main effect of Task was found in the 2-way ANOVAs. 


\section{Stimuli, Materials, and Equipment}

These were identical to experiment 1 .

\section{Task and Procedure}

The protocol was identical to experiment 1 with the following exceptions. Two additional block-sets were added with short foreperiods of $1200 \mathrm{~ms}$, one for each set of MI and MP instructions. The long and short foreperiod blocks were presented separately to minimise effects of mixing blocks with different foreperiods together. Second, a jittered intertrial interval of 1800-2200 ms was added between trials, and the response interval was fixed to $1000 \mathrm{~ms}$.

Due to the increased length of the experiment, participants attended in two sessions, each lasting approximately 40 minutes with a minimum interval of 24 hours separation. Both long and short foreperiod MP blocks were performed in the first session, and the long and short foreperiod MI blocks in the second session. Long and short foreperiod blocks were presented in counterbalanced order across participants, but consistent across sessions i.e., session 1 (long -> short), session 2 (long $\rightarrow$ short).

\section{Design and Data Analysis}

The analysis protocol followed a similar structure as in experiment 1 . Training trial data was not analysed. Trials with incorrect responses, or RTs faster than $100 \mathrm{~ms}$ were not included in the RT analysis (data removed in MI-Long $=3.16 \%$, MI-Short $=3.09 \%$, MP-Long $=2.72 \%$ MP-Short $=2.25 \%$ ). Priming effects for the response time and error rate congruency effects, costs, and benefits, and the mean neutral RTs, were analysed using separate two-way ANOVAs, with factors Task (MI, MP) and Duration (Long, Short), and post-hoc paired t-test comparisons were conducted where appropriate.

\section{Results}

\section{Imagery Ability}


Participants mean kinesthetic imagery ability score was $5.84 \pm 0.79$, and visual score was $4.51 \pm 0.97$.

Figure $3 \mathrm{~A}$ shows the mean reaction times for congruent, neutral, and incongruent trial types and for each MI and MP conditions, for each long and short foreperiod duration

\section{Figure 3}

All plots are divided into the two foreperiod Duration conditions, Long (3350 ms) and Hand (1200 ms). A: Mean reaction times (ms) for each trial type (congruent (Con), neutral (Neu), incongruent (Inc)) separated for the motor imagery (MI) and motor preparation (MP) conditions. B: Violin plots to visualise individual RT differences in the distribution of congruency effects $(C E)$ for each condition. Dot and triangle markers represent individual participants. Dashed lines represent participants with larger CEs for the MI condition compared to the MP condition, and solid lines represent a smaller CE in the MI compared to the MP condition. C: Mean cost and benefit effects (ms) for the MI and MP conditions. D: Mean error rates (\%) for each trial type (congruent, neutral, incongruent) separated for the MI and MP conditions. Error bars represent $\pm 1 S E M$. 
3A: Response Time

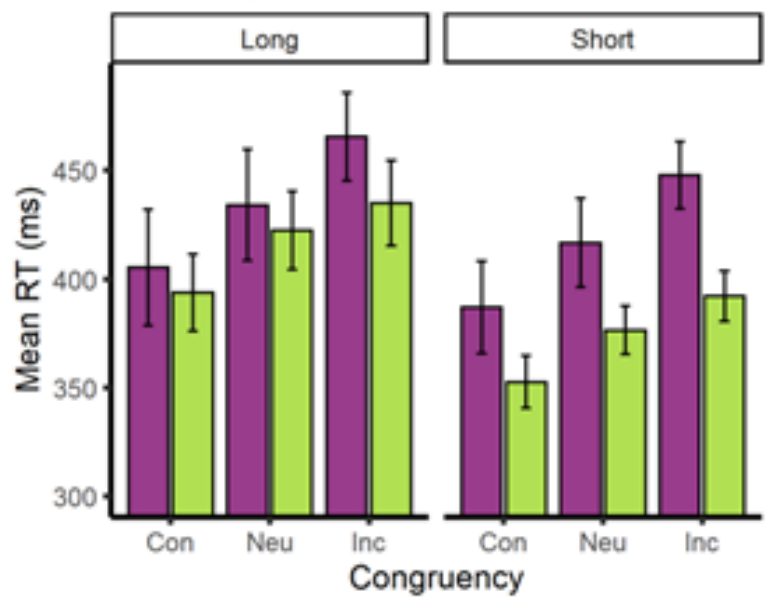

3C: Benefit and Cost Components

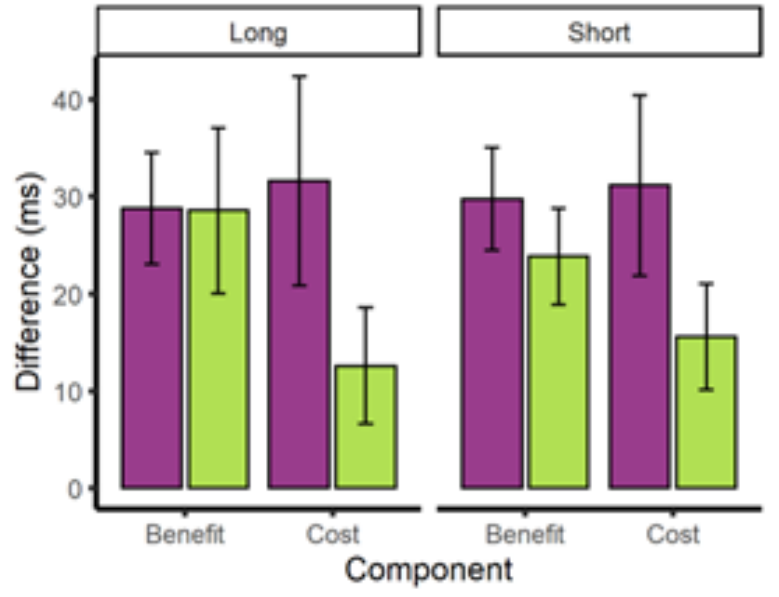

3B: Congruency Effects

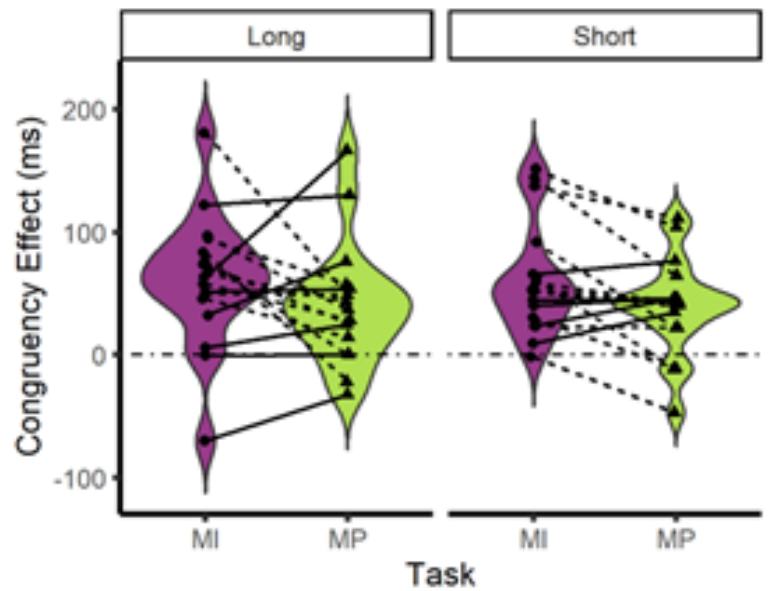

3D: Error Rates

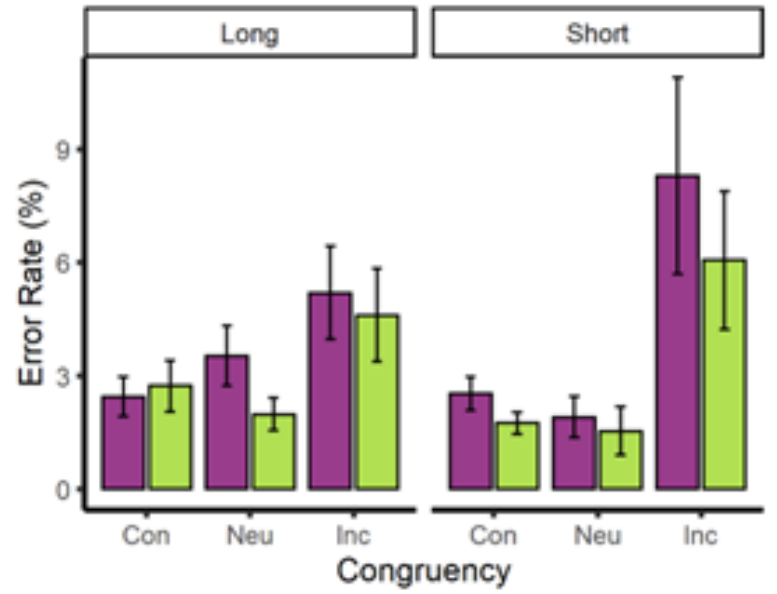

\section{Reaction Time Analysis}

\section{Neutral RTs}

The main effect of task was not significant $(F(1,16)=2.1564, \mathrm{p}=.132)$, meaning that reaction times were similar for the MI condition ( $425 \pm 22.00 \mathrm{~ms})$ and the MP condition $(399 \pm 13.50 \mathrm{~ms})$. However, the main effect of duration was significant $\left(F(1,16)=6.135, \mathrm{p}=.025, \eta^{2}=.277\right)$. showing that reaction times to the imperative stimulus were faster for the short duration condition $(396 \pm$ $14.60 \mathrm{~ms})$ compared to the long duration condition $(428 \pm 20.03 \mathrm{~ms})$. This effect was further modulated by task, as shown by the significant interaction between both factors $(F(1,16)=7.400$, $\mathrm{p}=.015 \eta^{2}=.316$ ). More specifically, mean reaction time differences between the MI and MP conditions were enhanced for the short duration (MP: $377 \pm 11.14 \mathrm{~ms}$; MI: $417 \pm 20.48 \mathrm{~ms}$ ) compared to the long duration (MP: $434 \pm 25.63 \mathrm{~ms}$; MI: long: $422 \pm 17.96 \mathrm{~ms}$ ). 


\section{Congruency Effects}

As seen in Figure 3B, the MI congruency effect was larger than the MP effect $(61 \pm 10.71 \mathrm{~ms}$ vs $40 \pm 10.05 \mathrm{~ms})$. This difference was supported by a significant main effect of task $(\mathrm{F}(1,16)=$ $\left.5.316, \mathrm{p}=.035, \eta^{2}=.249\right)$. The $\mathrm{t}$-test confirmed significance $(\mathrm{t}(16)=2.31, \mathrm{p}=.034, \mathrm{dz}=0.56)$, but the effect size came under our sensitivity threshold of 0.72 , suggesting possible unreliability of the effect. Neither the main effect of duration $(F<1)$ nor the interaction was significant $(F<1)$, suggesting the increased MI priming effect did not depend on differences in temporal uncertainty.

\section{Benefit}

As seen in Figure 3C, benefits were present for the MI $(29 \pm 4.95 \mathrm{~ms})$ and the MP conditions (26 $\pm 6.24 \mathrm{~ms})$. However, the two-way ANOVA revealed no significant main effects of task or duration and no significant interaction between both factors (all $\mathrm{F}<1$ ).

\section{Cost}

In contrast to benefits, the cost effect analysis showed a significant main effect of task $(F(1$, 16) $=5.906, p=.027)$ showing that the costs, which when compared using a t-test against zero were present in both MI $(31 \pm 8.72 \mathrm{~ms}, \mathrm{t}(16)=3.600, \mathrm{p}=.002)$ and the MP conditions $(14 \pm 5.04 \mathrm{~ms}$; $\mathrm{t}(16)=2.799, \mathrm{p}=.013)$, were still significantly larger for the MI compared to the MP condition. Meanwhile neither main effect of duration $(\mathrm{F}<1)$ nor interaction between both factors were significant $(\mathrm{F}<1)$.

In sum, mean reaction times in the neutral condition were faster for the MP compared to the MI condition, most substantially in the short foreperiod blocks. More importantly, congruency effects were enhanced in the MI compared to the MP condition, which was driven by enhanced cost effects in the MI condition. Similar congruency, benefit and cost effects were found for the short and the long interval durations.

\section{Error Rate Analysis}


Mean error rates, plotted in Figure 3D, show a tendency for committing more errors in incongruent compared to congruent trials, and a slightly larger congruency effect in MI (4.26 \pm $1.49 \%)$ compared to MP $(3.10 \pm 1.33 \%)$. Besides this latter effect, reflected in a significant main effect of Task for the congruency effects, $\mathrm{F}(1,16)=6.502$, $\mathrm{p}=.016$ ), there were no other significant effects or interactions in any of the ANOVAs.

\section{Discussion}

In line with previous findings, mean RT was longer for long duration foreperiods (Niemi \& Näätänen, 1981), suggesting our manipulation of temporal uncertainty was successful. Additionally, we found substantial cost and benefit effects in both MI and MP conditions. Most importantly, even though the cost effect was present in MP blocks, MI priming effects were still larger than MP priming effects regardless of foreperiod duration. Thus, experiment 2 demonstrates that the absent MP cost effect of experiment 1 cannot explain the increased MI priming effect, and that it likely does not reflect a reduction in temporal uncertainty due to MI for longer foreperiods.

One unexpected result was the interaction between duration and task instructions when comparing the mean neutral RTs. One possible explanation for this effect is that inhibition during MI (Bart et al, 2020; Rieger et al, 2017) has more time to dissipate, or otherwise does not overlap with other sources of inhibition that occur during response preparation (e.g., Greenhouse et al, 2015), and thus is weaker with longer compared with shorter foreperiods.

Overall, the results of experiment 2 provided additional evidence in support of the initial finding, that MI priming effects are larger than MP priming effects, and moreover that the results are not dependent on differences in temporal uncertainty for long versus short foreperiods. One interpretation of this finding is that MI does not provide additional "when" parameter information about upcoming responses compared to MP but may yet enhance existing "what" parameter information.

\section{Experiment 3}


In experiment 3, we manipulated the effector system used for response, and the associated preparation and imagery preceding it, by asking participants to make left or right finger responses in a new set of blocks, in analogy to the foot movements used in experiments 1 and 2. Foot-response and imagery blocks were also used. The experimental design was otherwise identical to experiment 2 , with the exception that only the short foreperiods were used in all blocks of this experiment.

We expected foot responses to produce slower RTs than finger responses, in line with previous findings (Seashore \& Seashore, 1941). Additionally, we expected that if the larger MI priming effect was an artefact of the requirement to use the feet for imagery and response, then the increased MI priming effect would be limited to the lower limb effectors. Alternatively, if the larger MI priming effect is a more general feature of the motor system, it would be larger than MP priming in both hand and foot effectors.

\section{Method}

\section{Participants}

Eighteen people, who had not participated in experiment 1 or 2, consented to participate in this experiment. One individual was not included in the final analysis due to slow performance (mean RT greater than 2.5 standard deviations from the group mean in at least four trial types), therefore the final sample consisted of seventeen individuals aged 19 - 33 years old (Mean age $=23$ years; 15 female, 2 male). One individual was left-handed, according to the EHI, 15 participants were right foot dominant, and the remaining were neither left nor right foot dominant.

The sensitivity analysis was identical to experiment 2 , revealing a minimum reliably detectable Cohen's dz of 0.72 for the paired t-test comparisons between MI and MP congruency effects, assuming a main effect of Task was found in the 2-way ANOVAs.

\section{Task and Procedure}

The protocol was identical to experiment 2, with the following exceptions. Only short foreperiods were used for all trials and blocks. Foot response blocks were identical to the short MI 
and MP blocks described in experiment 2. Finger response block-sets were added to both conditions, requiring MP for, and MI of left or right index finger movements (informative trials), or of both index fingers simultaneously (uninformative trials), prior to making index finger responses on the $\mathrm{Z}$ and $\mathrm{M}$ keys on a QWERTY keyboard. Participants again took part in two sessions, with response effector blocks counterbalanced between participants but in consistent orders across sessions within participants. Instructions for finger response blocks were modified to emphasise the preparation, imagery and response using index fingers, rather than feet.

\section{Data Analysis}

The analysis procedure was identical to the previous experiment, except to replace the Duration factor with a Limb factor with levels Finger and Foot. Data removed in MI-Finger $=4.90 \%$, MI-Foot $=4.63 \%$, MP-Finger $=3.21 \%$ MP-Foot $=3.41 \%$.

\section{Results}

\section{Imagery Ability}

Participants mean kinesthetic imagery ability score was $6.02 \pm 0.56$, and visual score was $4.86 \pm 1.20$.

\section{Reaction Time Analysis}

Figure 4A shows the mean reaction times for congruent, neutral, and incongruent trial types and for each MI and MP conditions, for each effector system.

\section{Figure 4}

All plots are divided into the two Limb conditions, Foot (foot responses) and Hand (finger responses). A: Mean reaction times ( $m s)$ for each trial type (congruent (Con), neutral (Neu), incongruent $(\mathrm{Inc})$ ) separated for the motor imagery (MI) and motor preparation (MP) conditions. B: Violin plots to visualise individual RT differences in the distribution of congruency effects $(C E)$ for each condition. Dot and triangle markers represent individual participants. Dashed lines represent participants with larger CEs for the MI condition compared to the MP condition, and solid lines represent a smaller CE in the MI compared to the MP condition. C: Mean cost and benefit effects (ms) for the MI and MP 
conditions. D: Mean error rates (\%) for each trial type (congruent, neutral, incongruent) separated for the MI and MP conditions. Error bars represent \pm 1 SEM.
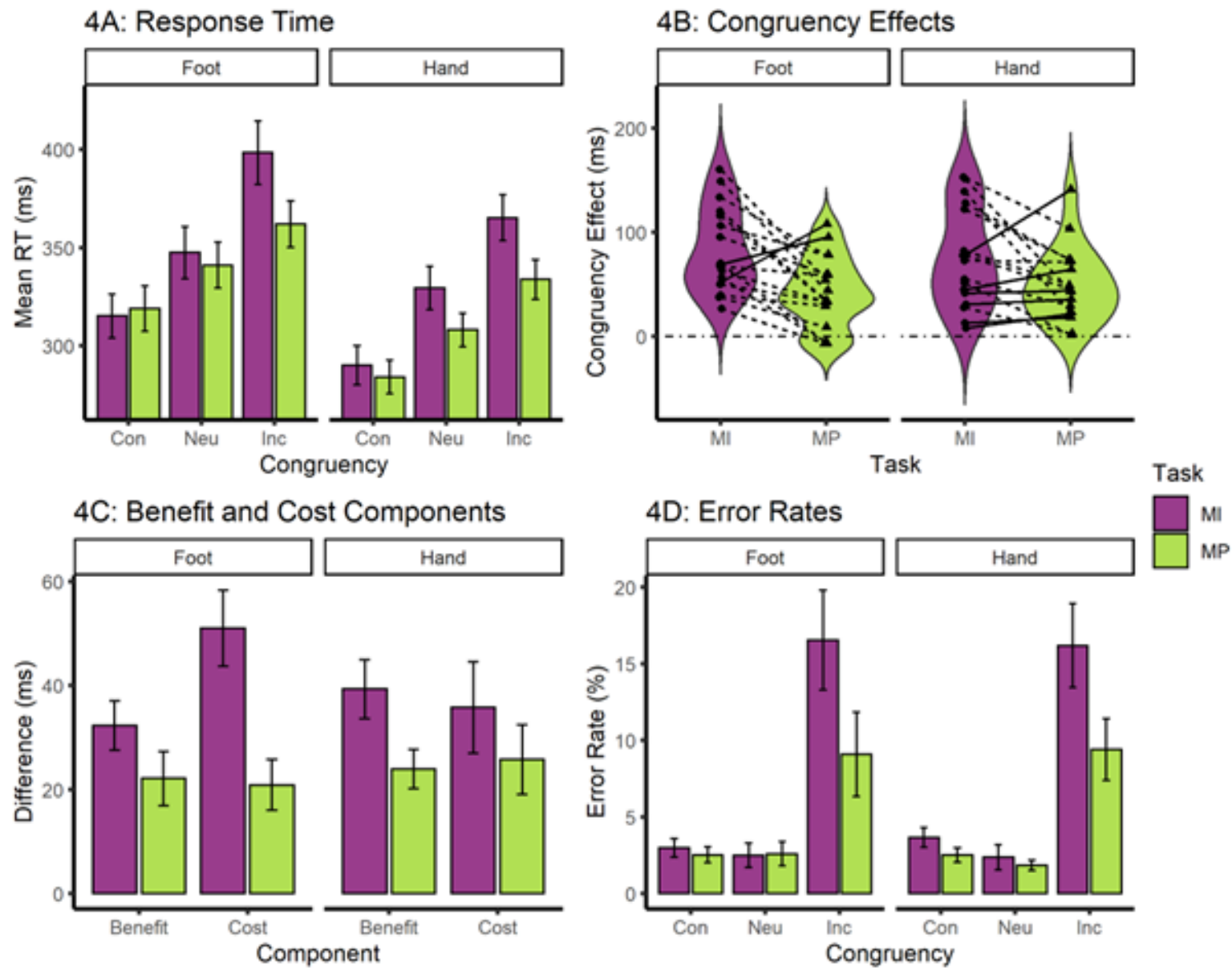

\section{Neutral Trials}

As seen in Figure 4A, mean RTs for the MI neutral trials (338 $\pm 9.60 \mathrm{~ms})$ were slower than MP neutral trials $(325 \pm 7.76 \mathrm{~ms})$. The main effect of task was significant $(\mathrm{F}(1,16)=5.909, \mathrm{p}=.027$, $\left.\eta^{2}=.27\right)$. The main effect of Limb was not significant $\left(F(1,16)=3.436, p=.082, \eta^{2}=.18\right)$, showing that reaction times to the imperative stimulus were similar for hand responses $(319 \pm 9.38 \mathrm{~ms})$ and foot responses $(344 \pm 11.95 \mathrm{~ms})$. The interaction between both factors was also not significant $(\mathrm{F}(1,16)=$ $\left.4.176, \mathrm{p}=.058, \eta^{2}=.207\right)$.

\section{Congruency Effects}


As seen in Figure 4B, congruency effects were larger in both effector systems for the MI (79 $\pm 10.45 \mathrm{~ms})$ than MP condition ( $36 \pm 4.67 \mathrm{~ms})$, which was supported by a significant main effect of $\operatorname{task}\left(\mathrm{F}(1,16)=13.741, \mathrm{p}=.002, \eta^{2}=.462\right)$. The $\mathrm{t}$-test confirmed significance $(\mathrm{t}(16)=3.071, \mathrm{p}=.002, \mathrm{dz}$ $=0.93$ ), and the effect size was above our expected sensitivity threshold of $d z=0.72$. Neither the Limb main effect nor interaction were significant (F's $<1.5)$, suggesting differences in congruency effect magnitude were similar for both effectors.

\section{Benefit}

A similar pattern of findings emerged when analysing the benefits, as seen in Figure 4C. Again, only the main effect of task was significant $\left(F(1,16)=9.929, p=.006, \eta^{2}=.383\right)$ showing that, although congruency effects were present for both the MI condition $(31 \pm 6.22 \mathrm{~ms})$ and the MP conditions ( $21 \pm 3.97 \mathrm{~ms}$ ), they were significantly larger for the MI compared to the MP condition. Again, neither the main effect of Limb nor interaction between both factors were significant $\left(F^{\prime} s<1.7\right)$.

\section{Cost}

Similar effects were found for costs. Again, only the main effect of task was significant $(\mathrm{F}(1$, $16)=8.593, \mathrm{p}=.010)$ showing that costs were significantly larger for the MI $(44 \pm 7.33 \mathrm{~ms})$ compared to the MP condition $(22 \pm 4.49 \mathrm{~ms})$. The main effect of $\operatorname{Limb}(F(1,16)=1.992, \mathrm{p}=.177)$ and the interaction between both factors were not significant $(\mathrm{F}(1,16)=3.737, \mathrm{p}=.071)$.

\section{Error Rate Analysis}

The mean error rate in the neutral condition was $2.44 \pm 0.76 \%$ for the MI task and $2.22 \pm$ $0.44 \%$ for the MP task, as can be seen in Figure 4D. However, the two-way ANOVA revealed no significant main effects or interactions (F's $<1)$. Regarding the priming effects in error rates, we observed a significant main effect only for the congruency (MI $13.03 \pm 2.50 \%$ vs MP $6.72 \pm 1.94 \%$; $\left.F(1,16)=13.135, p=.002, \eta^{2}=.451\right)$ and cost effect (MI $13.92 \pm 2.30 \%$ vs MP $7.02 \pm 1.97 \% ; F(1$, 16) $\left.=15.320, p=.001, \eta^{2}=.489\right)$, whereby MI effects were larger compared to MP effects. All other interactions and main effects were not significant (all F's $<1$ ). 


\section{Discussion}

In this experiment, participants were faster when responding with fingers than with feet, in line with previous findings (Seashore \& Seashore, 1941), although the effect was not significant. Furthermore, we observed larger MI priming effects compared to MP priming effects once again, and this was the case in both finger and foot response blocks. This suggests the increased MI priming effect observed in experiment 1 was not due to the novelty of requiring foot imagery and responses, and that the MI congruency effect increase may be a more general feature of the motor system.

\section{Experiment 4}

In experiments 1 to 3, we consistently observed larger response priming effects following MI compared to MP alone. However, as outlined in the introduction, recent research suggests MI is associated with a cognitive resource requirement (Glover \& Baran, 2017). Even though we have decided to analyse the congruency effects because it accounts for such baseline processing differences, it is plausible that MI is not increasing congruency effects compared to MP through genuine motoric influences. Rather, the increase in cognitive resources required by MI may interfere with ongoing MP, leading to an artificial increase of congruency effects.

However, when attention demanding tasks are interleaved between a precue and imperative stimulus, the beneficial effects of informative precues tend to be abolished (Eversheim \& Bock, 2002), preparation efficiency is reduced, and participants delay the onset of readying an action plan (Sangals \& Sommer, 2010). In these previous studies the interference of secondary tasks on main tasks was assessed based on general RT effects, rather than implementation and analysis of priming effects like those used in the present set of experiments. It is presently unknown whether secondary task interference would increase the congruency effect compared to MP alone, in the manner of an artificial inflation as proposed above or reduce them due to reduced preparation efficiency (Sangals \& Sommer, 2010).

To examine this possible cognitive load explanation for the enhanced MI congruency effect, experiment 4 introduced a third block-set which instructed participants to perform MP alongside a 
non-motor cognitive load task (hereafter cMP). First, we expected to be able to differentiate between 1) the "cognitive load inflation" hypothesis, which would predict larger congruency effects with an interleaved secondary task than MP alone, versus 2) the "reduced preparation efficiency" hypothesis, which would predict smaller priming effects for the interleaved task than MP. (Eversheim \& Bock, 2002; Sangals \& Sommer, 2010). Second, we intended to use this result to evaluate whether stronger MI priming is more due to cognitive load inflation, or a rather genuine response priming effect. Specifically, hypothesis 1) would predict the smallest priming effect for MP, and larger effects for both MI and cMP, whereas hypothesis 2) would predict the smallest priming effect for cMP, intermediate for MP, and largest for MI.

\section{Method}

\section{Participants}

Twenty-three people who had not participated in any of the previous experiment were recruited. One participant's dataset was removed due to slow responding (mean RT greater than 2.5 standard deviations from the group mean in at least two trial types). The final sample consisted of 22 participants aged $18-24$ years (mean age $=20$ years; 15 females, 7 male). One individual was left-handed, according to the EHI. Eighteen participants were right foot dominant, 2 were left foot dominant, 1 neither, nor 1 provided no response.

The sensitivity analysis was also based on a congruency effect comparison between MI and MP, revealing a minimum reliably detectable Cohen's dz of 0.61 with sample size 23 and power of 0.8 .

\section{Stimuli, Task and Procedure}

Stimuli were reduced in size in preparation for a separate EEG experiment using this task: (precue dimensions: $0.6^{\circ} \times 0.6^{\circ}$ visual angle, imperative dimensions: $0.6^{\circ} \times 0.3^{\circ}$ visual angle) Additional stimuli for the counting and motor preparation (cMP) condition were white outlines of star-shaped polygons with dimensions: $0.5^{\circ}$ x $0.5^{\circ}$ visual angle (see the last column of Figure $1 \mathrm{~A}$ ). 
The cMP instructions were the same as for MP, but additionally required participants to perform a secondary mental counting task, which was interleaved between the S1 and S2 stimuli in each trial. The counting task was designed with the following three assumptions about MI performance, to ensure the motor "cognitive load" was emulated in a non-motor task: First, MI performance depends on manipulation of information in working memory during the foreperiod (A1; e.g., Kraeutner et al, 2020). Second, MI performance is variable between individual trials (A2). Third, average MI performance quality is consistent throughout a block (A3). A counting task was designed in which participants made a mental running total of the number of stars presented, in vertical arrangement, on the same monitor used for the experimental task, during the foreperiod of each trial They reported the final total to the experimenter for each block. This design satisfied the assumptions as follows: a mental running total needed to be updated and maintained on each trial (A1). Variable numbers of stimuli were presented in each trial $(0,2$ or 3 ; A2), and this updating and maintenance of working memory was necessary across a whole block (A3).

The MI and MP task blocks were identical to the foot response blocks in Experiment 3, and the new cMP block-set was performed within the same session. They were performed in a fixed order, MP first and MI last, to prevent participants performing MI in the other blocks, and to layer instructions about the secondary task onto the already performed MP task. Responses, preparation, and imagery were performed with the feet only.

\section{Design and Data Analysis}

Training trial data was not analysed. Trials with incorrect responses, or RTs faster than 100 $\mathrm{ms}$ were not included in the RT analysis (data removed in $\mathrm{MP}=2.52 \%, \mathrm{cMP}=3.73 \%, \mathrm{MI}=4.70 \%$ ). Repeated-measures one-way 3-level ANOVAs using one Task factor with levels cMP, MP, and MI were used for the RTs and error rate congruency effect, benefit, cost and neutral trial data. Additionally, order-restricted Bayesian tests (Morey \& Wagenmakers, 2013) were used to evaluate the hypothesis that congruency effects, benefits and costs in cMP would be smallest, in MP intermediate, and in MI largest. Order-restricted tests compare the evidence in favour of a specific ordering of means versus a null model which considers all possible orderings, using Bayesian model 
comparison. This was conducted using the bayestestR package in R (Makowski et al, 2019).

Orthonormal contrasts were used, and the hypothesis comparison was specified using the string "cMP $<\mathrm{MP} \& \mathrm{MP}<\mathrm{MI}$ ". Bayes factors $(\mathrm{BF})$ of greater than 1 indicate support in favour of the alternative hypothesis, and less than 1 in favour of the null. More precisely, $\mathrm{BF}>3$ or $<1 / 3$ provides "substantial" evidence towards each of these respective hypotheses (Lee \& Wagenmakers, 2013).

\section{Results}

\section{Imagery Ability}

Participants mean kinesthetic imagery ability score was $6.12 \pm 0.56$, and visual score was $5.32 \pm 1.29$.

\section{Reaction Time Analysis}

The data for the RTs are plotted in Figure 5A as a function of task and congruency.

\section{Figure 5}

A: Mean reaction times ( $m s)$ for each trial type (congruent (Con), neutral (Neu), incongruent (Inc)) separated for the counting + motor preparation $(c M P)$, motor imagery (MI) and motor preparation $(M P)$ conditions. B: Violin plots to visualise individual RT differences in the distribution of congruency effects (CE) for each condition. Dot and triangle markers represent individual participants. Dashed lines represent participants with larger CEs for the MI condition compared to the $c M P$ or MP, respectively. Solid lines represent a smaller CE in the MI compared to the MP or cMP condition. C: Mean cost and benefit effects (ms) for the MI and MP conditions. D: Mean error rates (\%) for each trial type (congruent, neutral, incongruent) separated for the MI and MP conditions. Error bars represent \pm 1 SEM. 

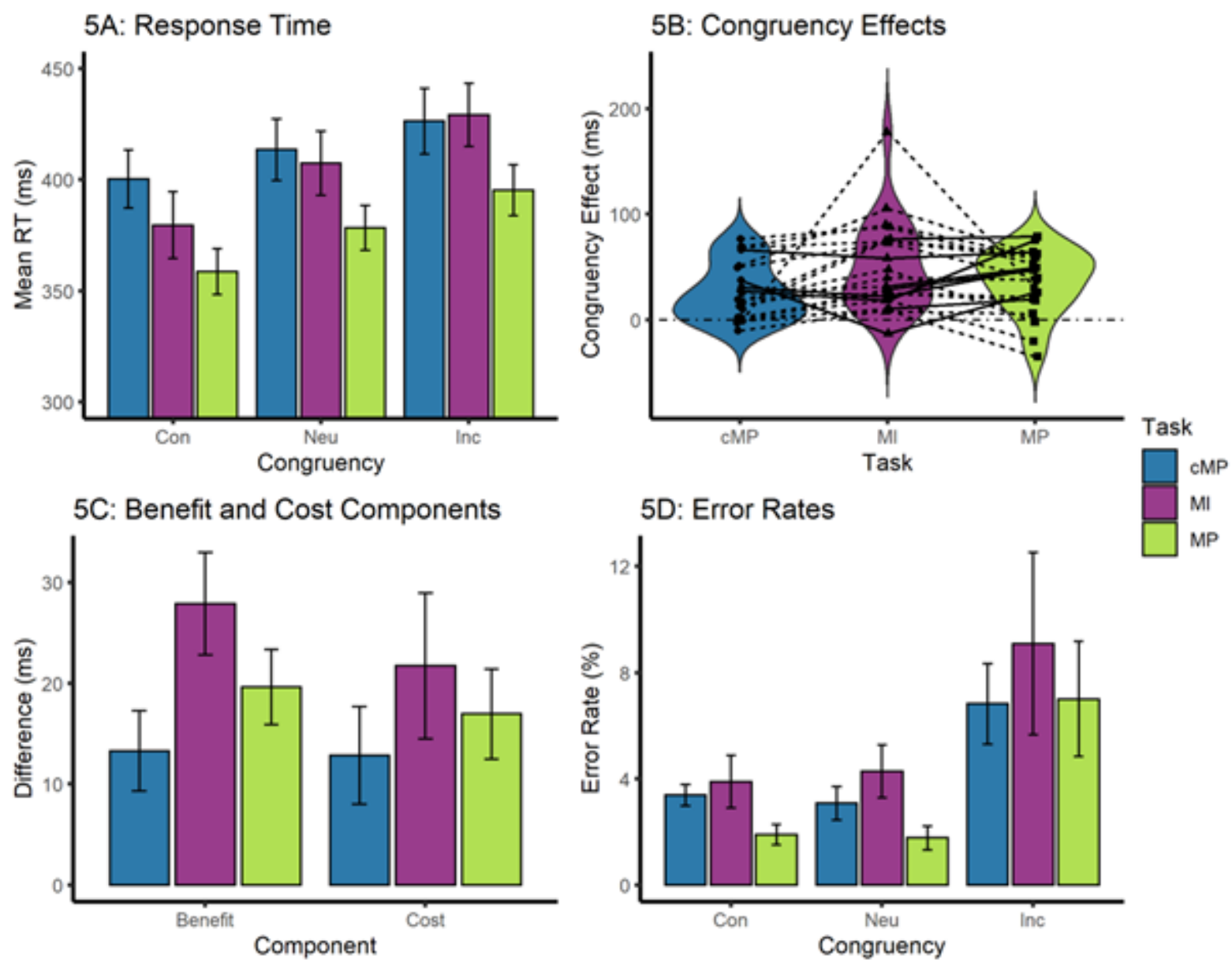

\section{Neutral Trials}

Mean neutral RTs were larger in MI (405 $\pm 8.65 \mathrm{~ms})$ and cMP conditions (413 $\pm 8.00 \mathrm{~ms})$, than in the MP condition ( $377 \pm 6.31 \mathrm{~ms})$, as can be seen in Figure 5A. This was confirmed with a significant main effect of task $\left(F(2,42)=7.68, p=.001, \eta^{2}=.268\right)$ and Bonferroni corrected post-hoc paired t-tests showing that reaction times in the MP condition were shorter than for the MI condition $(\mathrm{t}(21)=2.95, \mathrm{p}=.008)$ and the $\mathrm{cMP}(\mathrm{t}(21)=4.09, \mathrm{p}<.001)$. The reaction times were not different for the MI and cMP conditions $(t<1)$.

\section{Congruency effects}

The congruency effects matched our predictions of order, as can be seen in Figure 5B. They were largest in MI $(50 \pm 9.18 \mathrm{~ms})$, intermediate in MP $(37 \pm 6.39 \mathrm{~ms})$ and smallest in cMP $(26 \pm 5.71$ $\mathrm{ms})$, This was also reflected in the significant main effect of Task $\left(F(2,41)=4.26, p=.021, \eta^{2}=.169\right)$. However, the Bonferroni corrected post-hoc paired t-tests (alpha $=.016)$ revealed no significant 
differences between MI and cMP congruency effects $(\mathrm{t}(21)=2.55, \mathrm{p}=.019)$ nor MI and MP $(\mathrm{t}(21)=1.48, \mathrm{p}=.155)$, nor MP and $\mathrm{cMP}(\mathrm{t}(21)=1.85, \mathrm{p}=.079)$. However, the order restricted Bayesian test produced a $\mathrm{BF}_{10}$ of 4.36 , providing moderate evidence in favour of the hypothesis that congruency effects would be ordered with cMP lowest and MI highest, and MP as intermediate condition.

\section{Benefits}

A similar pattern of findings emerged when analysing the benefits, as can be seen in Figure 5C, also following the same ordering, with MI largest $(27 \pm 5.10 \mathrm{~ms})$, MP intermediate $(19 \pm 3.72 \mathrm{~ms}$, and cMP smallest $(13 \pm 3.96 \mathrm{~ms})$. These effects were significantly different between task types $(\mathrm{F}(2$, $\left.42)=4.32, p=.019, \eta^{2}=.170\right)$. Bonferroni corrected post-hoc paired t-tests (alpha $\left.=.016\right)$ showed significant differences between benefits in MI and $\mathrm{cMP}(\mathrm{t}(21)=2.62, \mathrm{p}=.016)$ but not $\mathrm{MP}(\mathrm{t}(21)=$ 1.82, $\mathrm{p}=.082)$, and no differences between MP and $\mathrm{cMP}(\mathrm{t}(21)=1.32, \mathrm{p}=.201)$. The order restricted Bayesian test produced $\mathrm{BF}_{10}$ of 4.88 , or moderate evidence in favour of the predicted ordering also for benefit effects, similar to congruency effects.

\section{Costs}

In contrast to the congruency and benefit effects, the cost effects (MI $=22 \pm 7.20 \mathrm{~ms} ; \mathrm{MP}=19$ $\pm 3.72 \mathrm{~ms} ; \mathrm{cMP}=13 \pm 3.96 \mathrm{~ms})$ were of similar magnitude for all three task types $(\mathrm{F}(2,42)=0.671$, $\mathrm{p}=.516$ ). In line with this analysis, the order restricted Bayesian test produced a $\mathrm{BF}_{10}$ of 2.88 , which is anecdotal evidence in favour of the predicted ordering of means for the costs.

\section{Error Rates Analysis}

\section{Neutral Trials}

As seen in Figure 5D, mean error rates in the neutral condition were largest in MI ( $4.28 \pm$ $0.99 \%)$, smallest in MP $(1.77 \pm 0.45 \%)$ and intermediate in cMP $(3.08 \pm 0.63 \%)$. This was reflected in a significant main effect of task type $\left(\mathrm{F}(2,42)=6.79, \mathrm{p}=.003, \eta^{2}=.244\right)$. Bonferroni corrected posthoc paired t-tests (alpha $=.016)$ showed that error rates in the MP condition were lower than for the 
MI condition $(\mathrm{t}(21)=3.22, \mathrm{p}=.004)$ and the $\mathrm{cMP}(\mathrm{t}(21)=3.29, \mathrm{p}=.003)$, but MI and cMP did not differ significantly $(t(21)=1.52, p=.145)$. No other significant differences of interest were observed for the congruency effects, benefits or costs in the error rate data (all F's $<1$ ).

\section{Discussion}

In this last experiment, participants were slower to respond in both MI and cMP conditions compared to MP conditions. The congruency effects were smallest in the cMP condition and largest in the MI conditions, with intermediate effects for the MP condition. These results provide evidence first that an interleaved attention task reduces the size of the congruency effect, in support of a reduced preparation efficiency hypothesis of priming effects (Sangals \& Sommer, 2010). Second, they show that the MI congruency effect cannot be explained as a form of motor-cognitive load.

\section{General Discussion}

In this study, we examined whether MI can be distinguished from MP in terms of the magnitude of their behavioural RT and error rate priming effects in subsequent real actions. This question was derived from a need to clarify a straightforward interpretation of the original FE hypothesis, as formulated by Jeannerod (1994), which suggests MI and MP should reflect the same motor plans but does not specify whether the relative informativeness (or value, reliability etc.) of these plans might differ. Although the original paper specified that protocols which examine the effects of $\mathrm{MI}$ on subsequent action can bring light to the FE hypothesis (Jeannerod, 1994, p. 191), direct comparisons between MI and MP to evaluate if the two behaviours can be distinguished or not under FE have remained relatively untested. On the basis of certain previous empirical (e.g. Anwar et al, 2011) and theoretical literature (e.g. O’Shea \& Moran, 2017), in this paper we proposed MI involves more informative motor plans than MP, and thus should produce larger behavioural priming effects - in other words, we proposed a strict interpretation of the FE hypothesis may not hold. In the first of four experiments, we obtained preliminary evidence in favour of our alternative hypothesis, 
whereby priming responses through MI resulted in larger overall congruency effects compared to MP. Crucially, by comparing the magnitude of mean RT priming effects instead of mean RTs themselves, we minimised the influence of baseline differences between the two conditions which had confounded interpretations of previous work (Ramsey et al, 2010; Li et al, 2004; 2009). In the experiments that followed, we obtained further evidence that this phenomenon cannot be explained by differences in temporal uncertainty between MI and MP conditions (experiment 2), is a general feature of the motor system, being present in both upper and lower limb responses (experiment 3) and cannot be explained as a form of motorcognitive load (experiment 4). Together, these results support the need to refine the original FE hypothesis regarding the degree to which MI and MP activate the same motor plans, to reflect the possibility that although the same plans are used by both, MI plans may be more informative for subsequent action.

Before proceeding to the main discussion, some limitations in our experiment warrant further consideration. As can be seen in Table 1, the effect sizes reported in two of the statistically significant MI versus MP congruency effect comparisons (experiments 1 and 2) were around the threshold of the minimum detectable effect sizes, as calculated with sensitivity power analyses. These minimum effect sizes are the smallest practical effects the study was sufficiently powered to detect. Particularly in the case of experiment 2 , where statistical significance of the difference was paired with smaller effect sizes than sensitivity analyses indicated, the design of the study was underpowered. In other words, the effect we observed in experiment 2 contributes less reliable information to our interpretation of our data which is that MI plans are more informative than MP plans. Additional studies with larger sample sizes are required to replicate this finding because these would be powered sufficiently to detect lower effect sizes. However, it is important to nonetheless acknowledge the numerical direction of each critical comparison between MI and MP congruency effects 
was consistent with our hypothesis in every experimental manipulation, suggesting cumulative evidence is in favour of a larger MI than MP priming effect - and more informative plans in the former than the latter.

Second, despite the specific ordering of MP and MI tasks, we were unable to exclude the possibility that participants might have explicitly imagined responses during the foreperiod of the MP blocks. However, we believe it unlikely that participants would have specifically imagined the precise button presses we instructed them to perform on every trial without prior instruction. Indeed, it would appear MI is generally detrimental for overall response times (see Figures 2-5A) and error rates (Figures 2-5D), which suggests MI might be a disadvantageous strategy when being instructed to perform fast and accurately. Overall, we do not believe this significantly compromises our result interpretations. In future research, exit surveys might be useful in terms of screening whether participants engaged in unnecessary MI and, if so, to what extent.

Previous studies which investigated how MI might influence subsequent behaviour included baseline confounds (Ramsey et al, 2010; Li et al, 2004; 2009) which limited interpretation of their effects. It could reasonably be argued that the stronger interference of MI on performance, compared with the weaker facilitation effects reported in those studies was due to the comparison between an MI condition which involved inhibitory processes (Guillot et al, 2012a), and a non-MI condition which did not. Thus, inhibitory signals produced during MI may have interfered with initiation of real subsequent performance, masking any true facilitatory priming effects following MI. Thus, those studies reported not a priming effect of MI, associated with the motor representations themselves, but rather an effect related to MI-centric inhibition of motor representations and the motor system. In the present study, neutral priming trials were used in both conditions, providing only "when" but not "what" information for the upcoming movement (Brass \& Haggard, 2008), and acted as a 
task-specific baseline, in which inhibition was accounted for through subtractive logic of computing priming effects. Furthermore, the neutral trials allowed us to break down the overall congruency effect into constituent benefit and cost effects, to further understand the nature of MI priming as relatively more facilitative or one more susceptible to interference. In contrast to the earlier studies, we found that MI can indeed facilitate subsequent movement relative to neutral trials, but that this benefit was not as large or consistent as the interference effect. As seen in Table 1, there was a tendency across experiments for a larger cost rather than benefit of MI relative to MP. This result expands on past work demonstrating priming effects of MI, by showing that subsequent action may be more sensitive to incorrect MI than incorrect MP. However, this asymmetry may also reflect a ceiling effect on performance due to the simple responses used, which may restrict observation of larger beneficial effects. Future work may seek to evaluate MI priming effects using more complex imagery and/or responses. Alternatively, training periods could be used to build up imagery ability and enhance its effectiveness. Furthermore, the small effect sizes and occasional non-significant difference between conditions warrants further replication and extension. 


\section{Table 1}

Congruency, cost, and benefit magnitudes across all six experimental manipulations, and per-experiment computed sensitivity threshold for Cohen's $d z$, and achieved Cohen's dz for the Congruency effect MI vs MP comparisons. Bold numbers

\begin{tabular}{|c|c|c|c|c|c|c|}
\hline Experiment & $\begin{array}{l}\text { Cost } \\
(\mathrm{ms})\end{array}$ & $\begin{array}{l}\text { Benefit } \\
(\mathrm{ms})\end{array}$ & $\begin{array}{l}\text { Congruency } \\
\text { Effect } \\
(\mathrm{CE} ; \mathrm{ms})\end{array}$ & $\begin{array}{l}\text { CE difference } \\
(\mathrm{MI}-\mathrm{MP} ; \mathrm{ms})\end{array}$ & $\begin{array}{l}\text { Sensitivity } \\
\text { Cohen's dz }\end{array}$ & $\begin{array}{l}\text { Obtained } \\
\text { Cohen's dz }\end{array}$ \\
\hline
\end{tabular}

\begin{tabular}{llcccccc}
\hline Experiment 1 & & & & & & & \\
& MI & $\mathbf{2 0}$ & 18 & 38 & $\mathbf{2 0}$ & 0.68 & 0.69 \\
& MP & -1 & 19 & 18 & &
\end{tabular}

\section{Experiment 2a:}

Long

$\begin{array}{lllll}\text { MI } & 31 & 29 & 60 & 19 \\ \text { MP } & 14 & 29 & 41 & \end{array}$

Experiment 2b:

Short

$\begin{array}{lllll}\text { MI } & \mathbf{3 1} & \mathbf{3 0} & 61 & \mathbf{2 1} \\ \text { MP } & 16 & 24 & 39 & \end{array}$

\begin{tabular}{|c|c|c|c|c|c|c|c|}
\hline \multicolumn{7}{|l|}{$\begin{array}{l}\text { Experiment 3a: } \\
\text { Finger }\end{array}$} & \\
\hline \multirow{5}{*}{$\begin{array}{l}\text { Experiment 3a: } \\
\text { Foot }\end{array}$} & MI & 36 & 39 & 75 & & \multirow{5}{*}{0.72} & \multirow{5}{*}{0.92} \\
\hline & MP & 26 & 24 & 50 & & & \\
\hline & & & & & & & \\
\hline & MI & 51 & 32 & 83 & \multirow{2}{*}{40} & & \\
\hline & MP & 21 & 22 & 43 & & & \\
\hline \multicolumn{8}{|l|}{ Experiment 4} \\
\hline & MI & 22 & 28 & 50 & \multirow{3}{*}{13} & \multirow{3}{*}{0.61} & \multirow{3}{*}{ n.s. } \\
\hline & $\mathrm{MP}$ & 17 & 20 & 37 & & & \\
\hline & cMP & 13 & 13 & 26 & & & \\
\hline
\end{tabular}

Note. $(\mathrm{ms})=$ milliseconds. $\mathrm{MI}=$ Motor Imagery. $\mathrm{MP}=$ Motor Preparation. $\mathrm{cMP}=$ Counting with Motor Preparation, $\mathrm{n} . \mathrm{s} .=$ non-significant. Cohen's dz effect sizes are calculated by t/sqrt(n) (Lakens, 2013). Bold numbers reflect priming effects larger for MI than MP.

Neutral priming trials also provided some insight into the possible inhibitory processes of MI. Since neutral trials prime subsequent responses with only “when”, but not also specific "what" motor parameters (Brass \& Haggard, 2008), they may represent (when compared to MP) a global inhibition effect (Guillot et al, 2012). In support of this, neutral MI 
trials were generally slower than neutral MP neutral trials across experiments. Note, however, this significant slowing effect was not present for the long foreperiod durations; that is neutral MI trials were not slower than neutral MP trials for long foreperiods in experiment 1 (indeed MI RTs were faster than MP RTs, although this was not a significant difference) and experiment 2, as evidenced by the significant task x duration interaction. One possible explanation is that global MI-inhibition is strong at the start of the long foreperiod but gradually decays as a function of time (Bart et al, 2021). Additional research which further interrogates the influence of foreperiod duration on RT differences between neutral MP vs neutral MI may provide additional insight into the time course of MI-related inhibition effects..

However, MI slowing relative to MP was consistent across experiments also for incongruent, but not for congruent trials (Figures 2-5A). Although this was not statistically analysed for reasons of brevity (the interested reader is referred to the full outputs in Supplementary Note 1), this pattern of results may also reflect effector-specific inhibition (Bart et al, 2020; Rieger et al, 2017). However, the present design was not designed specifically to tease apart these inhibitory effects, and it remains unclear whether the strength of priming of MI plans and MI-related inhibition of these plans are independent processes or not (Solomon et al, 2019). The present paradigm could be adapted to examine effector specific or general inhibition effects by using TMS-evoked motor potentials, or H-reflexes in MI-primed congruent and incongruent muscles.

Inhibition in MI has also been described as an inherent component of imagined action plans (Guillot et al, 2012a), and several studies which compare MI with action have shown weaker pre-movement neural activity for imagined compared to executed performance (Eagles et al, 2015; Kranczioch et al, 2009; 2010; Lebon et al, 2019; Solomon et al, 2019; Kraeutner et al, 2014), supporting this account. However, the present results support 
seemingly the opposite conclusion - that MI plans are enhanced in some way, not suppressed. This discrepancy may depend on differences in the how researchers characterise MI and their related experimental design choices, i.e., either being more similar to the planning stages of action (Jeannerod, 1994; Hanakawa, 2016), or more similar to action itself (Vogt, 1994; Solomon et al, 2019). It may be the case that the choice of paradigm restricts the questions that can be asked about MI, but also interpretation of results. For instance, comparing the effects of MI and MP on action reflects the position that MI and MP are more similar, whereas comparing MI and real actions directly, as in earlier work from our own group (Kranczioch et al, 2009), reflects the position that MI is more action-like and by extension less planning-like. We suggest that both paradigms can provide a unique perspective and a unique set of possible questions with which MI as a behaviour in its own right can be examined. Future conceptual and theoretical work is needed to examine similarities and differences between the two perspectives and methodological approaches.

There are some observations which nonetheless support our argument that planning in MI is not suppressed, and which are in keeping with a purist interpretation of the original FE hypothesis. For example, Hanakawa et al, (2008) reported that the dorsal premotor cortex (PMd) is more active during MI than action or planning alone, which may reflect stronger MI action plans (see also Hanakawa et al, 2003 and Nakayama et al, 2016, for evidence of a rostro-caudal gradient of imagery/planning-like activity versus action-like activity in premotor areas). Other research demonstrates that inhibitory influences of supplementary motor area on primary motor cortex suggest a post-planning cortical inhibition during MI (Kasess et al, 2008; Bajaj et al, 2015), rather than an inhibition inherent to the motor plan. Finally, a recent study by Monaco et al, (2020) found that while action plans and MI can be independently decoded from anterior intraparietal sulcus (aIPS) and PMd, cross-decoding is only possible in aIPS. This suggests aIPS stores a generalised representation of motor plans 
(Aflalo et al, 2015) common to MI and MP, and in line with FE between them (Jeannerod, 1994). In contrast, poor cross-decoding in PMd could reflect differences in the premotor activation patterns between MI and MP. Altogether, these data fit well with a conceptualisation that a common motor plan is represented in MI and MP, but which is more informative and not necessarily inhibited during MI.

The results of experiment 4 suggested MI priming does not reflect increased motorcognitive load. At first glance this contradicts Glover \& Baran's (2017; Glover et al, 2020) motor-cognitive model (MCM) of MI. The MCM was motivated by the finding that although MI performance generally adheres to biomechanical constraints of the body (de Lange et al, 2006; Kilteni et al, 2018), its duration is subject to under and overestimations relative to real action (Guillot et al, 2012b). Glover and colleagues (2017; 2020) conducted a series of wellcontrolled studies which provided support for this model, indicating that when motorcognitive load is high, such as when imagining grasping something slippery, MI duration is more delayed than overt action. Such compelling results highlight the importance of nonmotor components of MI performance (Dahm \& Rieger, 2016; Kraeutner, et al, 2020; Ingram et al, 2016). More precisely, however, the MCM argues that action and imagery bifurcate at the level of action monitoring and control, which follows the motor planning stage (for which FE in motor representations is assumed to apply; Glover \& Baran, 2017). We would argue that instead of contradictory findings, the present results rather complement the MCM by demonstrating this bifurcation occurs even earlier, at the level of motor representations. In contrast to the online control of MI, however, we propose the differences in motor representations per se do not seem to reflect a load-like process, as evidenced by the divergence in priming effects between the MI and cMP conditions in experiment 4.

Predictive processing frameworks could form a strong theoretical basis to explain the findings that MI priming effect elicit stronger motor planning than MP alone. Predictive 
coding theories (Rao \& Ballard, 1999; Lee \& Mumford, 1992; Friston, 2005) suggest that perception and action are causal inference processes, mediated by the minimisation of prediction errors which arise through the comparison of top-down predictions with bottom-up sensory information. In the motor system, predictions descend from motor cortex, creating prediction errors which are reported by alpha motor neurons. Movement, produced by spinal reflex arcs, minimise these motoric prediction errors. That is, top-down predictions in the motor system are de facto motor commands, and action occurs to resolve the resulting mismatch between expected and actual body state (Adams et al, 2013). In predictive coding both predictions and prediction-errors can be weighted by their (zero-sum) precision, i.e., their relevance to an organism's (hierarchical, generative) model of body and environment. If the prediction errors have low precision, sensory signals will have little impact on the internal model of the body or environment the organism creates (Feldman \& Friston, 2010; Brown et al, 2011). On the other hand, predictions with low precision place greater emphasis on the sensory information about the world for updating the internal model.

Predictive coding has previously been invoked to explain action observation, a phenomenon related to MI (Kilner et al, 2007). In social situations the intentions of others can be inferred when observing another person's movements. This is achieved by attenuating the precision of motor prediction errors (Friston, Mattout, Kilner, 2011), but preserving the precision of top-down motor predictions. Thus, causes (i.e., intentions) of the observed actions can be inferred without acting them out. Similar weighting mechanisms may also occur during performance of MI. For example, motor-evoked potentials, reflecting high-level motor system activation, are facilitated during MI, but H-reflexes, which reflect the activation of 1a spinal afferents, are abolished or attenuated (Kasai et al, 1997; see Grosprêtre et al, 2019, for a discussion). In other words, top-down commands are preserved while bottom-up signals are suppressed during MI, as would be expected under a predictive coding 
mechanism. Additionally, learning during MI (e.g. Mulder et al, 2004; Kim et al, 2017; Krauetner et al, 2020; Yoxon \& Welsh, 2020) may occur when internal models for action are updated using fictive prediction errors (Shadmehr, Smith, \& Krakauer, 2010) which are generated as a function of (motor) memory-retrieval (Barron et al, 2020).

Predictive coding may thus provide the basis for understanding how motor plans in MI and MP vary in richness: by modulating the precision for the top-down predictions. Increasing the precision of motor predictions (which are the plans/commands for action) reflects a change in the informativeness or richness of plans relative to MP. Although little work has directly examined this in the motor domain, a recent neuroimaging study has shown enhanced top-down neural connectivity during visual imagery compared to visual perception, suggesting visual imagery also involves enhanced visual sensory predictions (Dijkstra et al, 2017). Furthermore, one explanation of visual hallucinations in psychosis is that they result from "unreasonably" strong (precise) prior (top-down) visual predictions (Sterzer et al, 2018). In the context of motor priming, more precise predictions in MI would effectively increase the size of prediction errors when incorporated into the subsequent movement, relative to MP. This difference in the magnitude of prediction error signals would increase the size of behavioural priming effects for MI compared to MP alone (Anwar et al, 2011; see also Feldman \& Friston, 2011).

Interestingly, one influential framework for MI-based skill learning, the perceptualcognitive approach (Frank et al, 2014; 2016), could be integrated with the predictive coding account for motor imagery. That is, more detailed action representation structures produced after MI training might reflect increases in the informativeness of action plans over time. A predictive coding framework for MI may be suitable for understanding other MI-related phenomena, such as the observation that prior experience with a novel action can facilitate its learning after MI training (e.g., Mulder, 2004), or that the combination of MI and action 
observation (AOMI; Vogt et al, 2013) leads to better training outcomes than each behaviour individually (Eaves et al, 2016, for a review). These effects can both be understood as reflecting changes in the informativeness of the motor prediction for the respective motor representation.

To conclude, this series of studies rigorously examined an alternative position to the strong FE (Jeannerod, 1994); that being, although MI and MP might use the same underlying motor plans, MI plans have a greater impact on subsequent action in terms of their costly and beneficial priming effects We found evidence to support this hypothesis, and propose that predictive processing models of MI, such as Ridderinkhof \& Brass (2015) IMPPACT model, may provide the most valuable next steps towards a greater mechanistic understanding of imagined action as a behaviour which lies at the intersection of planning (Anwar et al, 2011), performance (Solomon et al, 2019), and control of action (Glover \& Baran, 2017; Glover et al, 2020). 


\section{References}

Adams, R. A., Shipp, S., \& Friston, K. J. (2013). Predictions not commands: active inference in the motor system. Brain Structure and Function, 218(3), 611-643.

Aflalo, T., Kellis, S., Klaes, C., Lee, B., Shi, Y., Pejsa, K., ... \& Andersen, R. A. (2015). Decoding motor imagery from the posterior parietal cortex of a tetraplegic human. Science, 348(6237), 906-910.

Anwar, M. N., Tomi, N., \& Ito, K. (2011). Motor imagery facilitates force field learning. Brain research, 1395, 21-29.

Bart, V. K., Koch, I., \& Rieger, M. (2020). Inhibitory mechanisms in motor imagery: disentangling different forms of inhibition using action mode switching. Psychological Research, 1-21.

Bart, V. K., Koch, I., \& Rieger, M. (2021). Decay of inhibition in motor imagery. Quarterly Journal of Experimental Psychology, 74(1), 77-94.

Bajaj, S., Butler, A. J., Drake, D., \& Dhamala, M. (2015). Brain effective connectivity during motorimagery and execution following stroke and rehabilitation. NeuroImage: Clinical, 8, 572-582.

Barron, H. C., Auksztulewicz, R., \& Friston, K. (2020). Prediction and memory: A predictive coding account. Progress in neurobiology, 192, 101821.

Brass, M., \& Haggard, P. (2008). The what, when, whether model of intentional action. The Neuroscientist, 14(4), 319-325.

Brown, H., Friston, K. J., \& Bestmann, S. (2011). Active inference, attention, and motor preparation. Frontiers in psychology, 2, 218.

Cisek, P., \& Kalaska, J. F. (2005). Neural correlates of reaching decisions in dorsal premotor cortex: specification of multiple direction choices and final selection of action. Neuron, 45(5), 801814. 
Dahm, S. F., \& Rieger, M. (2016). Cognitive constraints on motor imagery. Psychological research, 80(2), 235-247.

Debarnot, U., Louis, M., Collet, C., \& Guillot, A. (2011). How does motor imagery speed affect motor performance times? Evaluating the effects of task specificity. Applied cognitive psychology, 25(4), 536-540.

de Lange, F. P., Helmich, R. C., \& Toni, I. (2006). Posture influences motor imagery: an fMRI study. Neuroimage, 33(2), 609-617.

Dijkstra, N., Zeidman, P., Ondobaka, S., van Gerven, M. A., \& Friston, K. (2017). Distinct top-down and bottom-up brain connectivity during visual perception and imagery. Scientific reports, 7(1), 1-9.

Eagles, J. S., Carlsen, A. N., \& MacKinnon, C. D. (2015). Neural processes mediating the preparation and release of focal motor output are suppressed or absent during imagined movement. Experimental brain research, 233(5), 1625-1637.

Eaves, D. L., Riach, M., Holmes, P. S., \& Wright, D. J. (2016). Motor imagery during action observation: a brief review of evidence, theory, and future research opportunities. Frontiers in neuroscience, 10, 514.

Eversheim, U., \& Bock, O. (2002). The role of precues in the preparation of motor responses in humans. Journal of motor behavior, 34(3), 271-276.

Faul, F., Erdfelder, E., Lang, A. G., \& Buchner, A. (2007). G* Power 3: A flexible statistical power analysis program for the social, behavioral, and biomedical sciences. Behavior research methods, 39(2), 175-191.

Feldman, H., \& Friston, K. (2010). Attention, uncertainty, and free-energy. Frontiers in human neuroscience, 4, 215. 
Frank, C., Land, W. M., Popp, C., \& Schack, T. (2014). Mental representation and mental practice: experimental investigation on the functional links between motor memory and motor imagery. PloS one, 9(4), e95175.

Frank, C., Land, W. M., \& Schack, T. (2016). Perceptual-cognitive changes during motor learning: The influence of mental and physical practice on mental representation, gaze behavior, and performance of a complex action. Frontiers in psychology, 6, 1981.

Friston, K. (2005). A theory of cortical responses. Philosophical transactions of the Royal Society B: Biological sciences, 360(1456), 815-836.

Friston, K., Mattout, J., \& Kilner, J. (2011). Action understanding and active inference. Biological cybernetics, 104(1), 137-160.

Glover, S., \& Baran, M. (2017). The motor-cognitive model of motor imagery: Evidence from timing errors in simulated reaching and grasping. Journal of experimental psychology: human perception and performance, 43(7), 1359.

Glover, S., Bibby, E., \& Tuomi, E. (2020). Executive functions in motor imagery: support for the motor-cognitive model over the functional equivalence model. Experimental brain research, $1-14$.

Greenhouse, I., Sias, A., Labruna, L., \& Ivry, R. B. (2015). Nonspecific inhibition of the motor system during response preparation. Journal of Neuroscience, 35(30), 10675-10684.

Gregg, M., Hall, C., \& Butler, A. (2010). The MIQ-RS: a suitable option for examining movement imagery ability. Evidence-Based Complementary and Alternative Medicine, 7(2), 249-257.

Grosprêtre, S., Lebon, F., Papaxanthis, C., \& Martin, A. (2019). Spinal plasticity with motor imagery practice. The Journal of physiology, 597(3), 921-934.

Guillot, A., Di Rienzo, F., MacIntyre, T., Moran, A., \& Collet, C. (2012a). Imagining is not doing but involves specific motor commands: a review of experimental data related to motor inhibition. Frontiers in human neuroscience, 6, 247. 
Guillot, A., Hoyek, N., Louis, M., \& Collet, C. (2012b). Understanding the timing of motor imagery: recent findings and future directions. International Review of Sport and Exercise Psychology, 5(1), 3-22.

Hétu, S., Grégoire, M., Saimpont, A., Coll, M. P., Eugène, F., Michon, P. E., \& Jackson, P. L. (2013). The neural network of motor imagery: an ALE meta-analysis. Neuroscience \& Biobehavioral Reviews, 37(5), 930-949.

Hanakawa, T. (2016). Organizing motor imageries. Neuroscience research, 104, 56-63.

Hanakawa, T., Dimyan, M. A., \& Hallett, M. (2008). Motor planning, imagery, and execution in the distributed motor network: a time-course study with functional MRI. Cerebral cortex, 18(12), 2775-2788.

Hanakawa, T., Immisch, I., Toma, K., Dimyan, M. A., Van Gelderen, P., \& Hallett, M. (2003). Functional properties of brain areas associated with motor execution and imagery. Journal of neurophysiology, 89(2), 989-1002.

Hardwick, R. M., Caspers, S., Eickhoff, S. B., \& Swinnen, S. P. (2018). Neural correlates of action: Comparing meta-analyses of imagery, observation, and execution. Neuroscience \& Biobehavioral Reviews, 94, 31-44.

Ingram, T. G., Solomon, J. P., Westwood, D. A., \& Boe, S. G. (2019). Movement related sensory feedback is not necessary for learning to execute a motor skill. Behavioural brain research, 359, 135-142.

Jeannerod, M. (1994). The representing brain: Neural correlates of motor intention and imagery. Behavioral and Brain sciences, 17(2), 187-201.

Jeannerod, M. (2001). Neural simulation of action: a unifying mechanism for motor cognition. Neuroimage, 14(1), S103-S109. 
Kasess, C. H., Windischberger, C., Cunnington, R., Lanzenberger, R., Pezawas, L., \& Moser, E. (2008). The suppressive influence of SMA on M1 in motor imagery revealed by fMRI and dynamic causal modeling. Neuroimage, 40(2), 828-837.

Kasai, T., Kawai, S., Kawanishi, M., \& Yahagi, S. (1997). Evidence for facilitation of motor evoked potentials (MEPs) induced by motor imagery. Brain research, 744(1), 147-150.

Kilner, J. M., Friston, K. J., \& Frith, C. D. (2007). Predictive coding: an account of the mirror neuron system. Cognitive processing, 8(3), 159-166.

Kilteni, K., Andersson, B. J., Houborg, C., \& Ehrsson, H. H. (2018). Motor imagery involves predicting the sensory consequences of the imagined movement. Nature communications, 9(1), 1-9.

Kim, T., Frank, C., \& Schack, T. (2017). A systematic investigation of the effect of action observation training and motor imagery training on the development of mental representation structure and skill performance. Frontiers in Human Neuroscience, 11, 499.

Kraeutner, S. N., Eppler, S. N., Stratas, A., \& Boe, S. G. (2020). Generate, maintain, manipulate? Exploring the multidimensional nature of motor imagery. Psychology of Sport and Exercise, 48, 101673.

Kraeutner, S., Gionfriddo, A., Bardouille, T., \& Boe, S. (2014). Motor imagery-based brain activity parallels that of motor execution: evidence from magnetic source imaging of cortical oscillations. Brain research, 1588, 81-91.

Kraeutner, S. N., Stratas, A., McArthur, J. L., Helmick, C. A., Westwood, D. A., \& Boe, S. G. (2020). Neural and behavioral outcomes differ following equivalent bouts of motor imagery or physical practice. Journal of Cognitive Neuroscience, 32(8), 1590-1606.

Kranczioch, C., Mathews, S., Dean, P. J., \& Sterr, A. (2009). On the equivalence of executed and imagined movements: evidence from lateralized motor and nonmotor potentials. Human brain mapping, 30(10), 3275-3286. 
Kranczioch, C., Mathews, S., Dean, P., \& Sterr, A. (2010). Task complexity differentially affects executed and imagined movement preparation: evidence from movement-related potentials. PloS one, 5(2), e9284.

Korka, B., Schröger, E., \& Widmann, A. (2021). The encoding of stochastic regularities is facilitated by action-effect predictions. Scientific Reports, 11(1), 1-14.

Lakens, D. (2013). Calculating and reporting effect sizes to facilitate cumulative science: a practical primer for t-tests and ANOVAs. Frontiers in psychology, 4, 863.

Lebon, F., Ruffino, C., Greenhouse, I., Labruna, L., Ivry, R. B., \& Papaxanthis, C. (2019). The neural specificity of movement preparation during actual and imagined movements. Cerebral Cortex, 29(2), 689-700.

Lee, T. S., \& Mumford, D. (2003). Hierarchical Bayesian inference in the visual cortex. JOSA A, 20(7), 1434-1448.

Lee, M. D., \& Wagenmakers, E. J. (2014). Bayesian cognitive modeling: A practical course. Cambridge university press.

Leuthold, H. (2003). Programming of expected and unexpected movements: Effects on the onset of the lateralized readiness potential. Acta psychologica, 114(1), 83-100.

Leuthold, H., Sommer, W., \& Ulrich, R. (2004). Preparing for action: inferences from CNV and LRP. Journal of psychophysiology, 18(2-3), 77.

Li, S., Latash, M. L., \& Zatsiorsky, V. M. (2004). Effects of motor imagery on finger force responses to transcranial magnetic stimulation. Cognitive Brain Research, 20(2), 273-280.

Li, S., Stevens, J. A., \& Rymer, W. Z. (2009). Interactions between imagined movement and the initiation of voluntary movement: a TMS study. Clinical Neurophysiology, 120(6), 11541160. 
Lorey, B., Bischoff, M., Pilgramm, S., Stark, R., Munzert, J., \& Zentgraf, K. (2009). The embodied nature of motor imagery: the influence of posture and perspective. Experimental brain research, 194(2), 233-243.

Makowski, D., Ben-Shachar, M. S., \& Lüdecke, D. (2019). bayestestR: Describing effects and their uncertainty, existence and significance within the Bayesian framework. Journal of Open Source Software, 4(40), 1541.

Morey, R. D., \& Wagenmakers, E. J. (2014). Simple relation between Bayesian order-restricted and point-null hypothesis tests. Statistics \& Probability Letters, 92, 121-124.

Monaco, S., Malfatti, G., Culham, J. C., Cattaneo, L., \& Turella, L. (2020). Decoding motor imagery and action planning in the early visual cortex: overlapping but distinct neural mechanisms. Neuroimage, 218, 116981.

Mulder, T., Zijlstra, S., Zijlstra, W., \& Hochstenbach, J. (2004). The role of motor imagery in learning a totally novel movement. Experimental brain research, 154(2), 211-217.

Nakayama, Y., Yamagata, T., \& Hoshi, E. (2016). Rostrocaudal functional gradient among the predorsal premotor cortex, dorsal premotor cortex and primary motor cortex in goal-directed motor behaviour. European Journal of Neuroscience, 43(12), 1569-1589.

Niemi, P., \& Näätänen, R. (1981). Foreperiod and simple reaction time. Psychological bulletin, 89(1), 133.

Oldfield, R. C. (1971). The assessment and analysis of handedness: the Edinburgh inventory. Neuropsychologia, 9(1), 97-113.

O’Shea, H., \& Moran, A. (2017). Does motor simulation theory explain the cognitive mechanisms underlying motor imagery? A critical review. Frontiers in human neuroscience, 11, 72.

Posner, M. I. (1980). Orienting of attention. Quarterly journal of experimental psychology, 32(1), 325. 
Ramsey, R., Cumming, J., Eastough, D., \& Edwards, M. G. (2010). Incongruent imagery interferes with action initiation. Brain and Cognition, 74(3), 249-254.

Rao, R. P., \& Ballard, D. H. (1999). Predictive coding in the visual cortex: a functional interpretation of some extra-classical receptive-field effects. Nature neuroscience, 2(1), 79-87.

Ridderinkhof, K. R., \& Brass, M. (2015). How kinesthetic motor imagery works: a predictiveprocessing theory of visualization in sports and motor expertise. Journal of PhysiologyParis, 109(1-3), 53-63.

Rieger, M., Dahm, S. F., \& Koch, I. (2017). Inhibition in motor imagery: a novel action mode switching paradigm. Psychonomic bulletin \& review, 24(2), 459-466.

Rosenbaum, D. A. (1980). Human movement initiation: specification of arm, direction, and extent. Journal of Experimental Psychology: General, 109(4), 444.

Sangals, J., \& Sommer, W. (2010). The impact of intervening tasks on response preparation. Journal of Experimental Psychology: Human Perception and Performance, 36(2), 415.

Seashore, S. H., \& Seashore, R. H. (1941). Individual differences in simple auditory reaction times of hands, feet and jaws. Journal of Experimental Psychology, 29(4), 342.

Shadmehr, R., Smith, M. A., \& Krakauer, J. W. (2010). Error correction, sensory prediction, and adaptation in motor control. Annual review of neuroscience, 33, 89-108.

Solomon, J. P., Kraeutner, S. N., Bardouille, T., \& Boe, S. G. (2019). Probing the temporal dynamics of movement inhibition in motor imagery. Brain research, 1720, 146310.

Sterzer, P., Adams, R. A., Fletcher, P., Frith, C., Lawrie, S. M., Muckli, L., ... \& Corlett, P. R. (2018). The predictive coding account of psychosis. Biological psychiatry, 84(9), 634-643.

Vogt, S. (1994). Imagery needs preparation too. Behavioral and Brain Sciences, 17(2), 226-227.

Vogt, S., Di Rienzo, F., Collet, C., Collins, A., \& Guillot, A. (2013). Multiple roles of motor imagery during action observation. Frontiers in human neuroscience, 7, 807. 
Yoxon, E., \& Welsh, T. N. (2020). Motor system activation during motor imagery is positively related to the magnitude of cortical plastic changes following motor imagery training. Behavioural Brain Research, 390, 112685. 